This article has been accepted for publication in Monthly Notices of the Royal Astronomical Society C: 2019 The Authors Published by Oxford University Press on behalf of the Royal Astronomical Society. All rights reserved. 


\title{
Particle re-acceleration and Faraday-complex structures in the RXC J1314.4-2515 galaxy cluster
}

\author{
C. Stuardi ${ }^{\oplus}, 1,2 \star$ A. Bonafede,${ }^{1,2}$ D. Wittor, ${ }^{1,2,3}$ F. Vazza ${ }^{\oplus},{ }^{1,2}$ A. Botteon ${ }^{\oplus},{ }^{1,2,4}$ \\ N. Locatelli, ${ }^{1,2}$ D. Dallacasa, ${ }^{1,2}$ N. Golovich, ${ }^{5}$ M. Hoeft,${ }^{6}$ R. J. van Weeren, ${ }^{4}$ \\ M. Brüggen ${ }^{3}$ and F. de Gasperin ${ }^{3}$ \\ ${ }^{1}$ Dipartimento di Fisica e Astronomia, Università di Bologna, via Gobetti 93/2, I-40122 Bologna, Italy \\ ${ }^{2}$ INAF - Istituto di Radioastronomia di Bologna, Via Gobetti 101, I-40129 Bologna, Italy \\ ${ }^{3}$ Hamburger Sternwarte, Universität Hamburg, Gojenbergsweg 112, D-21029 Hamburg, Germany \\ ${ }^{4}$ Leiden Observatory, Leiden University, PO Box 9513, NL-2300 RA Leiden, the Netherlands \\ ${ }^{5}$ Lawrence Livermore National Laboratory, 7000 East Avenue, Livermore, CA 94550, USA \\ ${ }^{6}$ Thüringer Landessternwarte Tautenburg, Sternwarte 5, D-07778 Tautenburg, Germany
}

Accepted 2019 August 22. Received 2019 August 22; in original form 2019 May 1

\begin{abstract}
Radio relics are sites of electron (re)acceleration in merging galaxy clusters but the mechanism of acceleration and the topology of the magnetic field in and near relics are yet to be understood. We are carrying out an observational campaign on double relic galaxy clusters starting with RXC J1314.4-2515. With Jansky Very Large Array multiconfiguration observations in the frequency range $1-4 \mathrm{GHz}$, we perform both spectral and polarization analyses, using the rotation measure (RM) synthesis technique. We use archival XMM-Newton observations to constrain the properties of the shocked region. We discover a possible connection between the activity of a radio galaxy and the emission of the eastern radio relic. In the northern elongated arc of the western radio relic, we detect polarized emission with an average polarization fraction of $31 \%$ at $3 \mathrm{GHz}$ and we derive the Mach number of the underlying X-ray shock. Our observations reveal low levels of fractional polarization and Faraday-complex structures in the southern region of the relic, which point to the presence of thermal gas and filamentary magnetic field morphology inside the radio emitting volume. We measured largely different RM dispersion from the two relics. Finally, we use cosmological magnetohydrodynamical simulations to constrain the magnetic field, viewing angle, and to derive the acceleration efficiency of the shock. We find that the polarization properties of RXC J1314.4-2515 are consistent with a radio relic observed at $70^{\circ}$ with respect to the line of sight and that the efficient re-acceleration of fossil electrons has taken place.
\end{abstract}

Key words: acceleration of particles - magnetic fields - polarization-radiation mechanisms: non-thermal - shock waves - galaxies: clusters: individual: RXC J1314.4-2515.

\section{INTRODUCTION}

During cluster mergers, the kinetic energy of in-falling materials is injected in the intracluster medium (ICM). A fraction of the dissipated energy could amplify the magnetic field and (re)accelerate particles (see e.g. Brunetti \& Jones 2014, for a review) leading to the formation of diffuse synchrotron emission, observable in the radio band. Extended radio sources on the Mpc scale, without any optical counterpart and with low surface brightness (i.e. $\sim 0.1-1 \mu \mathrm{Jy}$ $\operatorname{arcsec}^{-2}$ ), have been detected in some merging galaxy clusters (see e.g. van Weeren et al. 2019, and references therein). They are termed radio haloes and radio relics and they reveal the presence of $\sim \mu \mathrm{G}$ magnetic fields and $\sim \mathrm{GeV}$ relativistic particles in galaxy clusters.

Radio haloes permeate the central volume of most dynamically disturbed galaxy clusters. They are typically circular, with sizes $\geq 1$ Mpc, with low/absent polarization, down to a few per cent level (see e.g. Feretti et al. 2012, for a review). Radio haloes have a steep spectrum $^{1}$ with $\alpha>1$. Their presence in merging galaxy clusters suggests that the energy for particle (re)acceleration could come

\footnotetext{
${ }^{1}$ Hereafter, the spectrum is defined as $S_{v} \propto v^{-\alpha}$, where $S_{v}$ is the radio flux density at the frequency $v$ and $\alpha$ is the spectral index.
} 
from the gravitational energy released in the ICM during the process of structure formation (i.e. via turbulence; Cassano et al. 2010). The details of the acceleration mechanism are yet to be understood.

Radio relics are arc-shaped sources on the Mpc scale, located at the periphery of some merging galaxy clusters. They are characterized by a steep spectrum (i.e. $\alpha>1$ ) and strong polarization ( $\sim 20-50$ per cent at $1.4 \mathrm{GHz}$ ). According to simulations, during cluster mergers, shock waves move outwards along the merger axis and form pairs of symmetric radio relics that extend in the direction perpendicular to the merger axis (Brüggen et al. 2012; Ha, Ryu \& Kang 2018). Therefore, they are best observed when the merger occurs in the plane of the sky (Golovich et al. 2018, 2019). Although there is evidence that their origin is connected to shock waves generated in the ICM by merger events (Ensslin et al. 1998; Roettiger, Burns \& Stone 1999), the underlying particle acceleration mechanism is still under debate. The mechanism of diffusive shock acceleration (DSA) has been proposed (Bell 1978; Jones \& Ellison 1991): in this scenario, cosmic ray protons and electrons should be accelerated from the thermal pool up to relativistic energies by cluster merger shocks. Although this mechanism can explain the general properties of relic emission, several observational features remain unexplained:

(i) The observation of low Mach number shocks. Shock waves can be detected in the X-rays as sharp surface brightness discontinuities associated with a density jump. To date, a number of shock fronts have been detected using radio relics as shock tracers (Finoguenov et al. 2010; Akamatsu \& Kawahara 2013; Urdampilleta et al. 2018, for some collections). Typical Mach numbers $(M)$ of mergers shocks inferred from X-ray observations are between 1.5 and 3, with some exceptions at $M>3$ (Markevitch et al. 2002; Botteon et al. 2016b; Dasadia et al. 2016). In this regime, the electron acceleration efficiency predicted by the DSA model is at most of a few per thousands of the shock injected energy flux.

(ii) The non-detection of $\gamma$-ray emission in merging galaxy clusters. Protons are also expected to be accelerated by merger shocks and to produce $\gamma$-rays in the interaction with the thermal gas. The most updated Fermi upper limits (Ackermann et al. 2014) lead to a shock acceleration efficiency for protons even lower than what is normally assumed (i.e. below $10^{-3}$, Vazza \& Brüggen 2014; Vazza et al. 2016).

(iii) The high radio power of radio relics. The shock acceleration efficiency allowed by the DSA mechanism is not enough to match the radio emission observed in most radio relics (e.g. Markevitch et al. 2005; Botteon et al. 2016a; Eckert et al. 2016a; Hoang et al. 2018).

(iv) The radio spectral index of some relics. Clear cases in which spectral indices are difficult to reconcile with particle acceleration models are Abell 2256 (van Weeren et al. 2012) and the Toothbrush radio relic (van Weeren et al. 2016).

Additional mechanisms have been proposed, as for example the re-acceleration of pre-existing low-energy relativistic electrons (e.g. Pinzke, Oh \& Pfrommer 2013; Kang \& Ryu 2015, 2016). The origin of such fossil particles could be either in old remnants of active galactic nuclei (AGNs) or in an electron population accelerated by earlier shock waves. In this scenario, only a fraction of clusters may have adequate seed particle population and, in that case, the acceleration efficiency required to match radio observations would be lower. Furthermore, if the seed population of the AGN origin was mostly composed of electrons, the non-detection of gammaray emission would also be explained. Unfortunately, up to date, the connection between AGNs and radio relics could be established only in a few cases (e.g. Bonafede et al. 2014; van Weeren et al. 2017a).

Recent models have focused on the role of magnetic fields, that, in particular configurations, could allow electrons to reach suprathermal energies via the shock drift acceleration mechanism (SDA). Using particle in cell simulations, Guo, Sironi \& Narayan (2014a,b) have shown that, in a quasi-perpendicular pre-shock magnetic field (i.e. when the magnetic field lines are almost perpendicular to the shock normal, hence aligned with the shock front), electrons can also be pre-accelerated by shocks with $M=3$. At the same time, Caprioli \& Spitkovsky (2014) demonstrated that for quasiperpendicular shocks the proton acceleration is quenched for $M=$ 5. Although recent studies have confirmed that this might reduce the tension with the upper limits set by the Fermi collaboration (Wittor, Vazza \& Brüggen 2017), the role of the magnetic field and its amplification by low Mach number shocks is still poorly constrained.

To this end, obtaining highly resolved information on the polarization of radio emission in relics has the potential of revealing the local topology of magnetic fields in the electron cooling region. This is possible through the technique of rotation measure (RM) synthesis (Brentjens \& de Bruyn 2005).

We started the first systematic study of the magnetic field in radio relics. Our final goal is to constrain the magnetic field strength and structure in a sample of galaxy clusters, where shock waves that have been detected in X-ray and/or radio relics are observed. This will be possible by comparing values of RM derived from background sources in the pre-shock and post-shock regions with magnetic field models as in Bonafede et al. (2013). The full sample is made of 14 galaxy clusters with double radio relics observed with the Karl G. Jansky Very Large Array (JVLA) (Stuardi et al. in preparation).

Spectro-polarimetric receivers of the JVLA allow us to simultaneously study continuum and polarized emission with a $\mathrm{MHz}$ resolution at $\mathrm{GHz}$ frequencies. We can therefore perform the RM synthesis and study the polarized emission in galaxy clusters in a large range of frequencies and with a high resolution in the Faraday space. At the same time, JVLA allows us to study the diffuse radio emission in galaxy clusters on a variety of angular scales and at different frequencies while keeping the same resolution. Highresolution spectral index images are important to obtain information on the life cycle of the relativistic electrons that power radio sources (see e.g. van Weeren et al. 2017b)

The combined information on (i) magnetic fields at relics through radio polarimetric study, (ii) discovery of a possible connection between radio relic emission and AGN activity through highresolution spectral index imaging, and (iii) new detection of merger shocks through X-ray data analysis are the key ingredients to solve the problem of particle acceleration in low Mach number shocks.

In this paper, we study the radio emission of the galaxy cluster RXC J1314.4-2515 that belongs to our sample of double relics systems. We decided to focus on this galaxy cluster since it shows a number of interesting features: although it is a double relic cluster (i.e. the merger axis is expected to be on the plane of the sky) different works found that a significant component of the merger could lie along the line of sight (Golovich et al. 2018; Wittman, Cornell \& Nguyen 2018); the central radio halo is spatially connected with the western relic making their nature ambiguous; and the eastern radio relic was suspected to host a radio galaxy (Feretti et al. 2005; Venturi et al. 2007).

In Section 2, we briefly review literature information about this galaxy cluster, and in Section 3 we describe the radio observations and data reduction techniques. In Section 4, we analyse the results 
Table 1. Properties of RXCJ1314.4-2515. Rows 1 and 2: J2000 celestial coordinates of the X-ray cluster centroid; Row 3: redshift, $z$; Row 4: X-ray luminosity in the energy band $0.1-2.4 \mathrm{keV}$; Row 5: estimate of the hydrostatic mass. References: (1) Piffaretti et al. (2011), (2) Valtchanov et al. (2002), (3) Planck Collaboration XXVII (2016).

\begin{tabular}{lcc}
\hline RA (J2000) & $13^{\mathrm{h}} 14^{\mathrm{m}} 28^{\mathrm{s}} .0$ & $(1)$ \\
Dec. (J2000) & $-25^{\circ} 15^{\prime} 41^{\prime \prime}$ & $(1)$ \\
$z$ & 0.247 & $(2)$ \\
$L_{\mathrm{X}(0.1-2.4 \mathrm{keV})}^{\mathrm{SZ}}$ & $9.9 \times 10^{44} \mathrm{erg} \mathrm{s}^{-1}$ & $(1)$ \\
$M_{500}^{\mathrm{SZ}}$ & $6.7 \times 10^{14} \mathrm{M}_{\odot}$ \\
\hline
\end{tabular}

of continuum radio observations and discuss the spectral properties of the system. Polarization and RM synthesis studies are reported in Section 5. We discuss our results and conclude in Sections 6 and 7.

Throughout this paper we assume a $\Lambda$ cold dark matter cosmological model, with $H_{0}=69.6 \mathrm{~km} \mathrm{~s}^{-1} \mathrm{Mpc}^{-1}, \Omega_{\mathrm{M}}=0.286$, and $\Omega_{\Lambda}=0.714$ (Bennett et al. 2014). With this cosmology, 1 arcsec corresponds to $3.9 \mathrm{kpc}$ at the cluster redshift, $z=0.247$.

\section{RXC J1314.4-2515}

General information on this cluster is listed in Table 1. The radio contours obtained in Section 3 superimposed on optical and X-ray images are shown in Figs 1 and 2, respectively. RXC J1314.4-2515 shows two symmetric radio relics, east and west of the cluster. They were observed with the Very Large Array (VLA) at $1.4 \mathrm{GHz}$ (Feretti et al. 2005), and with the Giant Metrewave Radio Telescope (GMRT) at $610 \mathrm{MHz}$ (Venturi et al. 2007) and at $325 \mathrm{MHz}$ (Venturi et al. 2013). The western relic is more extended than the eastern one, and it is connected to a central radio halo. Recently, the galaxy cluster was observed also with the Murchison Widefield Array (MWA) from 88 to $215 \mathrm{MHz}$ (George et al. 2017), leading to an estimate of the integrated spectral index of eastern and western relics: $\alpha_{1.4 \mathrm{GHz}}^{118 \mathrm{MHz}}=1.03 \pm 0.12$ and $\alpha_{1.4 \mathrm{GHz}}^{118 \mathrm{MHz}}=1.23 \pm 0.09$, respectively.

RXC J1314.4-2515 has a disturbed morphology in the X-rays: it is elongated in the east-west direction, suggesting an ongoing merger activity along this axis (Valtchanov et al. 2002). In particular, Mazzotta et al. (2011) found that the western relic is coincident with a shock front, detected through XMM-Newton observations, with Mach $2.1 \pm 0.1$. They noticed that this shock front is M-shaped, with the nose of the front tilted inwards, which they proposed may be produced by the material in-falling along a filament. In the X-ray image, a sub-cluster in the south direction is also visible, with a stream of gas suggesting accretion by the northern main cluster (see Fig. 2).

Valtchanov et al. (2002) found a bi-modal distribution of the galaxies in this cluster both in velocity space $\left(\sim 1700 \mathrm{~km} \mathrm{~s}^{-1}\right.$ separation) and in projected space. This was recently confirmed by Golovich et al. (2018), who found also that the two merging sub-clusters have $\sim 1500 \mathrm{~km} \mathrm{~s}^{-1}$ line-of-sight velocity difference, suggesting that the merger axis has a substantial component along the line of sight. Recently, matching the observed projected separation and relative radial velocities between sub-clusters with cosmological $N$-body simulations, Wittman et al. (2018) constrained the angle between the sub-cluster separation vector and the line of sight. While in other double relics clusters the merger axis is found on the plane of the sky, for RXC J1314.4-2515 they obtained a maximum likelihood at $42^{\circ}$, although angles up to $90^{\circ}$ cannot be ruled out.

The median Galactic RM in the region of RXC J1314.4-2515 measured with an angular resolution of $8^{\circ}$ is $-30 \pm 2 \mathrm{rad} \mathrm{m}^{-2}$ (Taylor, Stil \& Sunstrum 2009). We used this value throughout the polarization analysis because we found the same median value outside the galaxy cluster in our field. This value is also consistent with the most updated estimate by Oppermann et al. (2012) and Hutschenreuter \& Enßlin (2019).

\section{DATA ANALYSIS}

\subsection{Radio observations}

The cluster has been observed with the JVLA in the $L$ band (1$2 \mathrm{GHz}$ ) in $\mathrm{A}, \mathrm{B}, \mathrm{C}$, and $\mathrm{D}$ configurations. These observations have a total bandwidth of $1024 \mathrm{MHz}$, subdivided into 16 spectral windows of $64 \mathrm{MHz}$ each (with 64 channels at frequency resolution of $1 \mathrm{MHz}$ ). We also reduced and analysed archival data in $S$ band (2-4 GHz) in DnC configuration, covering a total of $2048 \mathrm{MHz}$ in 16 spectral windows of $128 \mathrm{MHz}$ each (64 channels of $2 \mathrm{MHz}$ channel $^{-1}$ frequency resolution). Both data sets have full polarization products. Observing date, time, rms noise $(\sigma)$, and restoring beam of radio observations are listed in Table 2 .

\subsubsection{Calibration}

For calibration and total intensity imaging we used the CASA 5.3.0 package. We started the calibration process from data pre-processed by the VLA CASA calibration pipeline ${ }^{3}$ that performs basic flagging and calibration on Stokes $I$ continuum data. Then, we derived final delay, bandpass, gain/phase, leakage, and polarization angle calibrations and applied them to the target. The source 3C 286 was used as a bandpass, absolute flux density, and polarization angle calibrator for all the observations. We used the Perley \& Butler (2013) flux density scale and we followed the National Radio Astronomy Observatory polarimetry guide for polarization calibration. ${ }^{4}$ In particular, we performed a polynomial fit to the values of linear polarization fraction and angle tabulated in Perley \& Butler (2013) for 3C 286, to obtain a frequency-dependent polarization model. J1248-1959 was used as a phase calibrator for observations in A and B configurations in $L$-band, and for the $S$ band observations, while J1311-2216 was used for the observations in $\mathrm{C}$ and D configurations ( $L$ band). To correct for the instrumental leakage, an unpolarized source was used: J1407+2827 and 3C 147 for the $L$ - and $S$-band observations, respectively.

Radio frequency interference (RFI) was removed by statistical flagging algorithms also from the cross-correlation products. At the end of the flagging process, some spectral windows seriously affected by RFI were entirely removed. We flagged the frequency ranges: $2116-2244 \mathrm{MHz}$ in the $S$-band observations; from 1520 to $1584 \mathrm{MHz}$ in A configuration; from 1072 to $1136 \mathrm{MHz}$, and from 1520 to $1648 \mathrm{MHz}$ in B configuration; 1136-1264 and 1520$1584 \mathrm{MHz}$ in $\mathrm{C}$ configuration; from 1136 to $1328 \mathrm{MHz}$ and from 1520 to $1648 \mathrm{MHz}$ in D configuration. After RFI removal, we averaged the data sets in time down to $10 \mathrm{~s}$ to speed up the imaging process and we re-weighted the visibilities according to their scatter.

\footnotetext{
${ }^{2}$ https://casa.nrao.edu/

${ }^{3} \mathrm{https} / / /$ science.nrao.edu/facilities/vla/data-processing/pipeline

${ }^{4}$ https://science.nrao.edu/facilities/vla/docs/manuals/obsguide/modes/pol
} 


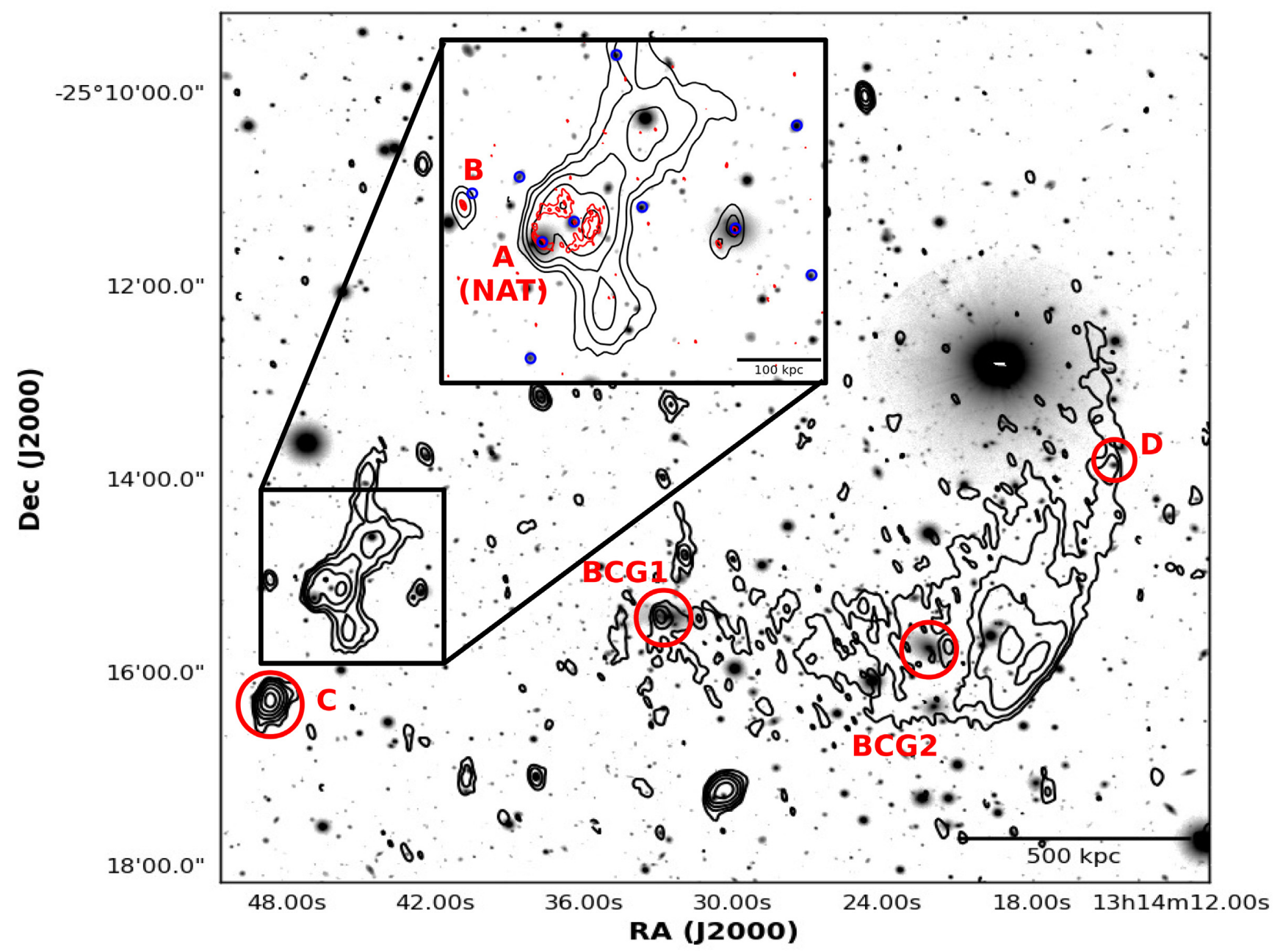

Figure 1. Subaru $r$ - and $g$-band image of the cluster RXC J1314.4-2515 with black radio contours at $1.5 \mathrm{GHz}$ overlaid. Contours are obtained combining $\mathrm{B}$ and $\mathrm{C}$ configurations, and the restoring beam is $9 \operatorname{arcsec} \times 5$ arcsec. Black contours start from $3 \sigma$, with $\sigma=0.015 \mathrm{mJy}^{-1}$ beam ${ }^{-1}$, and they are spaced by a factor of 2. A zoom in the region of the $\mathrm{E}$ radio relic is displayed in the top inset panel. Black contours are in $\mathrm{B}+\mathrm{C}$ configuration, same as above; red contours are from A configuration with a restoring beam of $2 \operatorname{arcsec} \times 1 \operatorname{arcsec}$, and they start at $3 \sigma$, with $\sigma=0.011 \mathrm{mJy}^{\text {beam }}{ }^{-1}$, spaced by a factor of 2 . Blue circles mark optically identified cluster members. Red letters and circles mark the sources with optical counterparts quoted in the paper. $B C G 1$ is the brightest cluster galaxy of the main sub-cluster; $B C G 2$ is the one of the western sub-cluster; A and B are cluster members; and C and D do not have redshift estimates (Golovich et al. 2018, 2019).

\subsubsection{Imaging and self-calibration}

We used the multiscale multifrequency de-convolution algorithm of the CASA clean (Rau \& Cornwell 2011) for wideband synthesisimaging. We set two terms for the Taylor expansion (nterms = 2) in order to take into account both the source spectral index (likely a power-law) and the primary beam response. We also used a $w$-projection algorithm to correct for the wide-field noncoplanar baseline effect (Cornwell, Golap \& Bhatnagar 2008) with an appropriate number of $w$-projection planes for each data set. We generally used the Briggs weighting scheme with the robust parameter set to 0.5 . We highlight in Table 2 the cases in which a different weighting scheme has been used.

There are two bright sources in the target field, one south-west of the cluster and the other to the north-east. The latter falls at the edge of the primary beam in $L$-band observations, causing problems for the self-calibration procedure. We set nterms $=3$ to individually image these sources. Then, we used the peeling technique to subtract them out of the images with direction-dependent gain solutions derived for each one. Some artefacts around the brightest source in the south are still present in the final images but their effect on the cluster emission is negligible. We used the peeling technique to subtract two variable sources before combining data at various configurations observed in different dates.

The combination of various antenna configurations differently flagged in frequency could in principle cause imaging artefacts due to uneven $u v$-coverage. To exclude the presence of strong artefacts we imaged each configuration individually before combining the different configurations.

Finally, cycles of self-calibration were performed to refine the antenna-based phase gain variations on the target field. The residual amplitude errors due to the calibration are estimated to be $\sim 5$ per cent. The local rms noise of the images is reported in Table 2. The final images were corrected for the primary beam attenuation using the widebandpbcor task in CASA. 


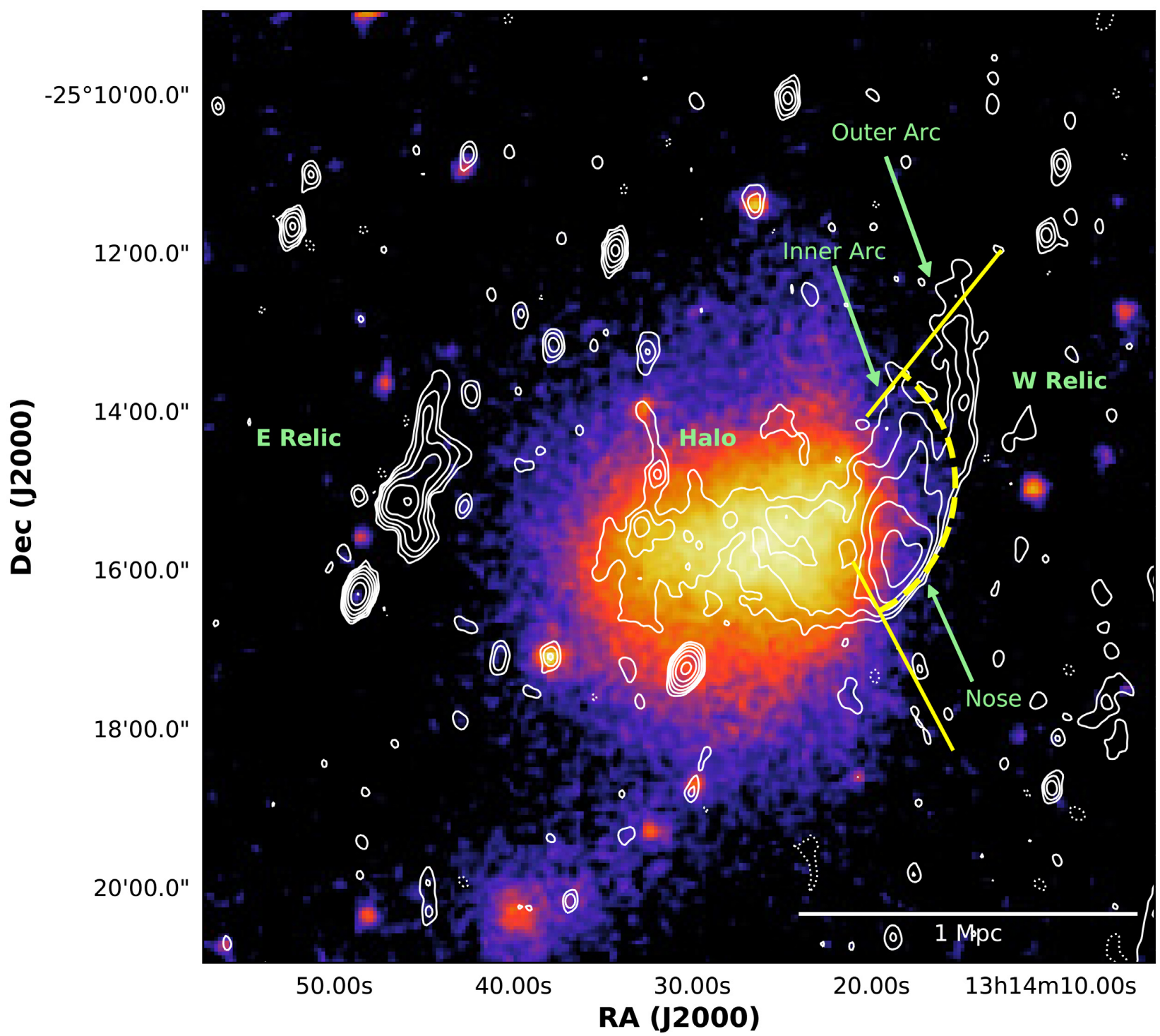

Figure 2. X-ray XMM-Newton image of the cluster RXC J1314.4-2515 with white radio contours overlaid. Contours are from $\mathrm{A}+\mathrm{B}+\mathrm{C}+\mathrm{D}$ configurations at $1.5 \mathrm{GHz}$ and start at $\pm 3 \times 0.016 \mathrm{mJy}_{\text {beam }}^{-1}$. They are spaced by a factor of 2 and negative contours are dotted. The restoring beam is 15 arcsec $\times 8$ arcsec. The X-ray image is smoothed with a Gaussian kernel of 5 arcsec. The yellow dashed line marks the position of the X-ray detected shock and the yellow sector encloses the region used to extract the surface brightness profile (see Section 6.2).

The $S$-band observations were performed on two pointings roughly centred on the east (E) and west (W) relic. We separately performed data reduction, peeling, and imaging of the two fields. Then, we joined the two final images correcting for the primary beam attenuation of both pointings.

In Fig. 1, radio contours at $1.5 \mathrm{GHz}$ in the combined $\mathrm{B}$ and $\mathrm{C}(\mathrm{B}+\mathrm{C})$ configurations are overlaid to the optical image of RXC J1314.4-2515 composite of Subaru $r$ and $g$ bands. A zoom of the E relic with A configuration high-resolution contours is also shown in Fig. 1. In Fig. 2, the radio contours obtained combining all the $L$-band observations $(\mathrm{A}+\mathrm{B}+\mathrm{C}+\mathrm{D})$ are overlaid on the $\mathrm{X}$ ray $X M M-N e w t o n$ image of the cluster. The $L$-band image obtained combining $\mathrm{C}$ and $\mathrm{D}(\mathrm{C}+\mathrm{D})$ configurations is shown in Fig. 3. The $S$-band image in DnC configuration is shown in Fig. 4.

\subsection{X-ray observations}

We retrieved from the XMM-Newton Science Archive two observations on RXC J1314.4-2515 (ObsID: 0501730101 and 0551040101), accounting for a total exposure time of $\sim 110 \mathrm{ks}$. The data sets were processed using the XMM-Newton Scientific Analysis System (SAS v16.1.0) and the Extended Source Analysis Software (ESAS) data reduction scheme (Snowden et al. 2008) following the working flow described by Ghirardini et al. (2019). We combined the count images and corresponding background and exposure maps of each ObsID to produce a single backgroundsubtracted image also corrected for the effects of vignetting and exposure time fluctuations. The image in the $0.5-2.0 \mathrm{keV}$ band is shown in Fig. 2. 
Table 2. Details of radio observations. Column 1: central observing frequency; Column 2: name of the frequency band; Column 3: array configuration; Column 4: date of the observation; Column 5: observing time; Column 6: robust parameter used for the Briggs weighting scheme (Briggs 1995) during imaging process; Column 7: size of the Gaussian taper used in the imaging process. If '-', no taper has been used; Column 8: FWHM of the major and minor axes of the restoring beam of the final image; Column 9: $1 \sigma$ rms noise of the total intensity image; Column 10: reference of the figures in this paper. Rms noise and beam shape of the images obtained with a combination of different configurations are reported under the horizontal line.

\begin{tabular}{|c|c|c|c|c|c|c|c|c|c|}
\hline $\begin{array}{l}\text { Freq. } \\
(\mathrm{GHz})\end{array}$ & Band & Array conf. & Obs. date & $\begin{array}{l}\text { Obs. time } \\
\text { (h) }\end{array}$ & Robust & Taper & Beam & $\begin{array}{l}\text { rms noise }(\sigma) \\
\left(\mathrm{mJy} \text { beam }^{-1}\right)\end{array}$ & Figure \\
\hline 1.5 & $L$ & A & 2018 Mar.-2018 Apr. & 5.5 & 0.5 & - & $\begin{array}{c}2 \operatorname{arcsec} \times \\
1 \operatorname{arcsec}\end{array}$ & 0.011 & 1 \\
\hline 1.5 & $L$ & B & 2017 Oct. & 2.0 & 0.5 & - & $\begin{array}{c}7 \operatorname{arcsec} \times \\
4 \operatorname{arcsec}\end{array}$ & 0.018 & \\
\hline 1.5 & $L$ & $\mathrm{C}$ & 2017 Jun. & 2.0 & 0.5 & - & $\begin{array}{c}24 \operatorname{arcsec} \times \\
11 \operatorname{arcsec}\end{array}$ & 0.035 & 9,10 \\
\hline 3.0 & $S$ & $\mathrm{DnC}$ & 2014 Sept. & 6.0 & 0.5 & - & $\begin{array}{c}17 \operatorname{arcsec} \times \\
13 \operatorname{arcsec}\end{array}$ & 0.012 & $4,9,10$ \\
\hline 1.5 & $L$ & $\mathrm{~B}+\mathrm{C}$ & & & 0.5 & - & $\begin{array}{c}9 \operatorname{arcsec} \times \\
5 \operatorname{arcsec}\end{array}$ & 0.015 & 1 \\
\hline 1.5 & $L$ & $\mathrm{C}+\mathrm{D}$ & & & 0.5 & - & $\begin{array}{c}25 \operatorname{arcsec} \times \\
11 \operatorname{arcsec}\end{array}$ & 0.014 & 3 \\
\hline
\end{tabular}

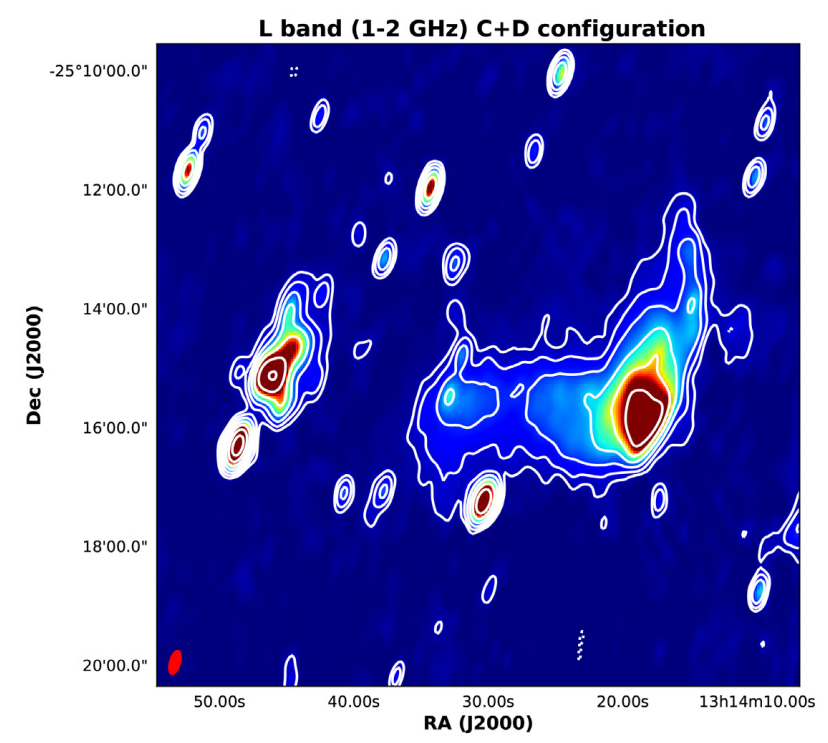

Figure 3. Lowest resolution image of the cluster in $\mathrm{C}+\mathrm{D}$ configuration at $1.5 \mathrm{GHz}$. White contours are overlaid, starting from $\pm 3 \sigma$, with $\sigma=0.014$ mJy beam ${ }^{-1}$, and they are spaced by a factor of 2 . Negative contours are dotted. The restoring beam of $25 \operatorname{arcsec} \times 11$ arcsec is shown in red in the left-hand corner and has a physical size of $\sim 70 \mathrm{kpc}$.

After the excision of contaminating point sources, we performed surface brightness and spectral analyses in the region of the western radio relic. The cluster emission was described with a thermal model with fix metallicity of $0.3 Z_{\odot}$ (e.g. Werner et al. 2013) and taking into account the Galactic absorption in the direction of the cluster as reported in Kalberla et al. (2005). The background was carefully treated by modelling both the astrophysical and instrumental components (see Ghirardini et al. 2019, for more details on the procedure).

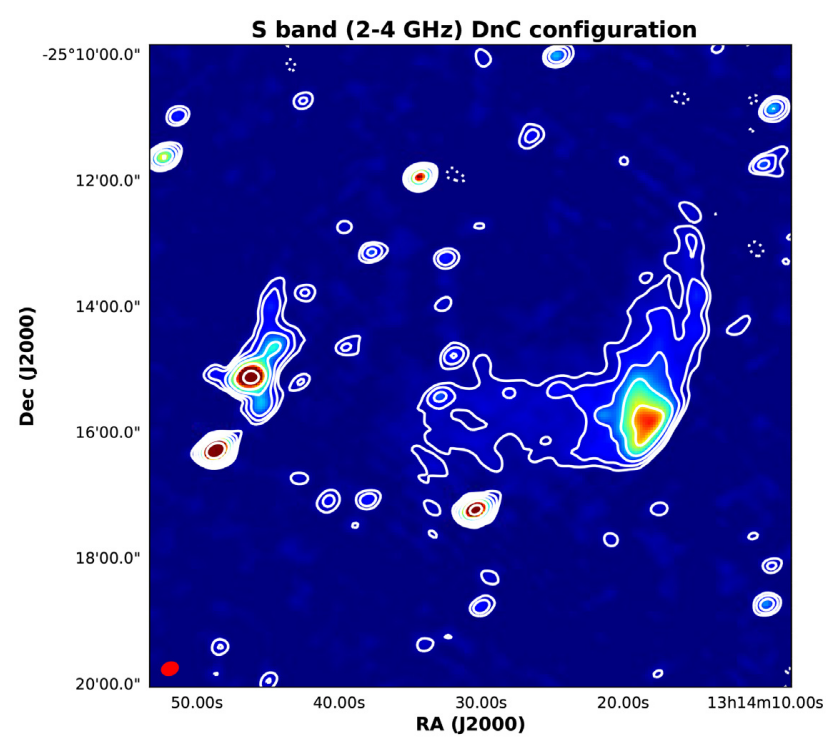

Figure 4. DnC configuration image at $3 \mathrm{GHz}$. White contours start by $\pm 3 \sigma$, with $\sigma=0.012 \mathrm{mJy}$ beam $^{-1}$, and they are spaced by a factor of 2 . Negative contours are dotted. The restoring beam is $17 \operatorname{arcsec} \times 13 \operatorname{arcsec}$ and it is shown in red at the left-hand corner. It has a physical size of $\sim 58 \mathrm{kpc}$.

\section{STUDY OF THE CONTINUUM EMISSION}

\subsection{Description of radio sources}

The eastern and western relics have a different shape but they are at about the same projected distance of $\sim 750 \mathrm{kpc}$ from the central brightest cluster galaxy of the main sub-cluster (BCG 1, at redshift $z=0.246$, see Fig. 1). A comparison between radio and X-ray images (Fig. 2) shows that the two relics are on the opposite sides of the cluster while the radio halo overlaps with the X-ray emission. There is a shift between the peak of X-ray surface brightness and 
the position of the BCG 1, as may be expected from an interacting system (Rossetti et al. 2016). The X-ray emission is elongated along the east-west merger axis and it is brighter on the western side of the cluster. At the position of the nose of the $\mathrm{W}$ relic, the $\mathrm{X}$-ray emission has a sharp drop where a shock was first detected by Mazzotta et al. (2011). We confirm and discuss the shock detection in Section 6.2. A stream of gas that follows the profile seems to connect the main cluster with a southern sub-cluster but in this region we did not detect any diffuse radio emission.

\subsubsection{The eastern relic}

The eastern relic has a largest linear size of $\sim 500 \mathrm{kpc}$. Its morphology and a plausible association with optical sources made Feretti et al. (2005) cautious about its identification with a radio relic. With high-resolution A configuration imaging we discovered that a narrow angle tail radio galaxy (NAT, e.g. Miley 1980) is embedded in the diffuse emission (marked with A in Fig. 1). This radio galaxy is a cluster member at redshift $z=0.242$ (Golovich et al. 2018, 2019). The diffuse emission is clearly related to the NAT but it extends well beyond radio galaxy lobes in the N-S direction and its largest size is perpendicular to the E-W merger axis. In Section 6.1, we discuss the relic-AGN connection using spectral index and polarization analyses. Another radio galaxy without redshift estimate ( $\mathrm{C}$ in Fig. 1) lies to the south of the $\mathrm{E}$ radio relic, at a projected distance of $\sim 200 \mathrm{kpc}$.

The $\mathrm{E}$ relic lies in a region of low X-ray surface brightness that prevents the possible detection of a shock related to the relic emission (see Fig. 2.)

\subsubsection{The western relic and the radio halo}

The dominant radio feature of RXC J1314.4-2515 is in the western part of the cluster. The faint diffuse emission of the halo with a roundish shape of radius $\sim 65 \operatorname{arcsec}$ (i.e. $250 \mathrm{kpc}$ ) is visible at the centre of the cluster in Fig. 2. The emission broadens and brightens to the west. Then it bends to the north and two arcs detach from the brightest region of the $\mathrm{W}$ relic along the $\mathrm{N}-\mathrm{S}$ direction. The innermost one extends for approximately $140 \mathrm{arcsec}$, corresponding to $\sim 550 \mathrm{kpc}$, and it is the brightest one. The outermost arc is more extended, reaching a largest linear size of $970 \mathrm{kpc}$ and a transverse size in the thinnest part of $\sim 80 \mathrm{kpc}$. The inner arc seems to follow the sharp X-ray profile in the west side of the cluster, while the longest one lies outside the region where the X-ray shock was detected. No clear optical counterpart could be associated with this radio emission. A point-like radio source without redshift estimate lies along the outermost arc (labelled with D in Fig. 1). All these features are observed also in the $S$-band image in Fig. 4.

\subsection{Spectral index study}

Using archival $S$-band data, we performed the spectral analysis of the extended emission to locate the site of particle acceleration.

We computed the spectral index between 1.5 and $3 \mathrm{GHz}$ based on the combined $L$-band $(\mathrm{B}+\mathrm{C}+\mathrm{D}$ in Table 2$)$ and $S$-band observations. We imaged the $L$-band observations with the same $u v$-range as the $S$ band data $(0.19-23.7 \mathrm{k} \lambda)$. We used the same pixel size and baseline interval, and set data weights in order to reach a similar beam size as in the $S$ band. After cleaning, we convolved the two images to the same Gaussian beam with full width at half-maximum (FWHM) of 18.5 arcsec and we corrected them for the primary beam response.
Table 3. Flux density, spectral index between 1.5 and $3 \mathrm{GHz}$ and radio power of extended sources measured from the images used for the spectral index study. We separated the region of the $\mathrm{W}$ relic and the halo on the basis of the spectral index profile as described in Section 4.2.2. The '*', stands for point-like sources subtracted.

\begin{tabular}{lcccc}
\hline Source & $\begin{array}{c}\text { Freq. } \\
(\mathrm{GHz})\end{array}$ & $\begin{array}{c}\text { Flux density } \\
(\mathrm{mJy})\end{array}$ & $\alpha_{3 \mathrm{GHz}}^{1.5 \mathrm{GHz}}$ & $\begin{array}{c}P_{1.4 \mathrm{GHz}} \\
\left(10^{24} \mathrm{~W} \mathrm{~Hz}^{-1}\right)\end{array}$ \\
\hline E relic & 1.5 & $11.3 \pm 0.6$ & $1.0 \pm 0.1$ & $2.3 \pm 0.1$ \\
& 3 & $5.6 \pm 0.3$ & & \\
W relic* & 1.5 & $33 \pm 2$ & $1.6 \pm 0.1$ & $7.9 \pm 0.5$ \\
& 3 & $10.9 \pm 0.8$ & & \\
Halo* $^{*}$ & 1.5 & $5.3 \pm 0.3$ & $1.3 \pm 0.2$ & $1.16 \pm 0.07$ \\
& 3 & $2.1 \pm 0.2$ & & \\
\hline
\end{tabular}

We have checked the position of a number of point-like sources in the two data sets to exclude any significant astrometric offset between them.

For the $\mathrm{W}$ relic and the halo, we computed the spectral index excluding point-like sources. We first imaged both $S$ - and $L$-band data sets excluding short baselines (i.e. $<3.5 \mathrm{k} \lambda$ ) that are sensitive to extended emission (i.e. larger than $90 \operatorname{arcsec}$ corresponding to $350 \mathrm{kpc}$ ). Then, we subtracted from the original visibilities the corresponding model components and we made new images using all the baselines at the resolution of $18.5 \mathrm{arcsec}$. This procedure was also applied to the E relic, but since the emission of the NAT is extended, it is impossible to properly separate the contribution of the tail from the relic. Hence, we decided not to subtract the radio galaxy. This choice allows us to study the spectral index behaviour from the core of the NAT to the lobes, and to investigate its connection with the diffuse source.

We computed the average flux density and spectral index of the E relic (and of the NAT radio galaxy) and of the $\mathrm{W}$ relic and the radio halo emission (sources subtracted) between 1.5 and $3 \mathrm{GHz}$. The values are reported in Table 3 . The uncertainties on the flux density measurements are computed as

$\sigma_{S}=\sqrt{(\delta S \times S)^{2}+\left(\sigma \times \sqrt{n_{\text {beam }}}\right)^{2}}$,

where $\delta S=5$ per cent is the calibration error, $\sigma$ is the rms noise listed in Table 2 and $n_{\text {beam }}$ is the number of beams in the sampled region. These uncertainties were then propagated to the spectral index.

We extrapolated the radio power at $1.4 \mathrm{GHz}$ from the $1.5 \mathrm{GHz}$ flux density measurement, considering a luminosity distance $D_{L}=$ 1253.3 Mpc (Wright 2006) and using the spectral indices for the $k$-correction. The uncertainties on flux densities were propagated to the radio power. The sum of the radio power of the two relics (sources subtracted) is consistent with the relation found by de Gasperin et al. (2014) between the radio power of double radio relics and the cluster mass.

Finally, we computed the spectral index for each pixel with value $>3 \sigma$ in both frequency bands. We show the spectral index map of the $\mathrm{E}$ relic in Fig. 5. We propagated the uncertainties on the flux densities pixel by pixel on the spectral index. The error map of the spectral index for the E relic is shown in the bottom panel of Fig. 5, while the spectral index image of the western region and its error map are shown in Fig. 6.

Spectral index images obtained from interferometric data should be treated with caution, since the value of $\alpha$ that we computed in each pixel is not independent of the neighbouring pixels. Furthermore, some artefacts (due to slightly different sampling of the 

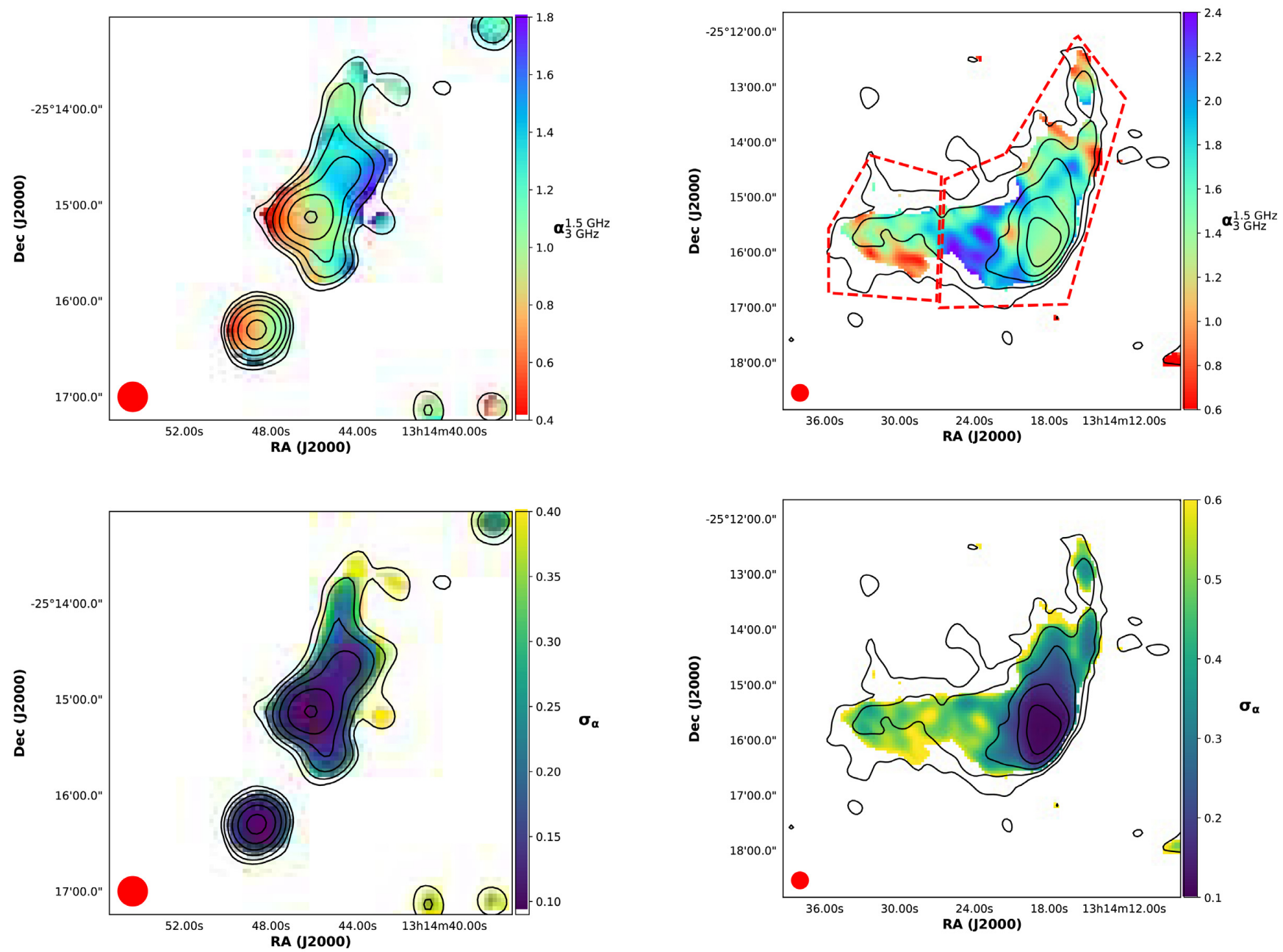

Figure 5. Top panel: Spectral index image of the E relic region between 1.5 and $3 \mathrm{GHz}$. The $1.5 \mathrm{GHz}$ total intensity contours $(\mathrm{B}+\mathrm{C}+\mathrm{D}$ configuration) are overlaid in black: the levels start at $3 \sigma$, with $\sigma=0.04 \mathrm{mJy} \mathrm{beam}^{-1}$, and are separated by a factor of 2. Bottom panel: Error map of the spectral index image with the same contours overlaid. The restoring beam of the two images is shown in the left-hand corner and its size is $18.5 \operatorname{arcsec} \times$ $18.5 \operatorname{arcsec}$

baselines and to imaging artefacts in the two original images) could affect spectral index images, in particular at the edge of extended emission or where point-like sources were subtracted out. We can recover the spectral index variations with higher significance by integrating the flux over regions larger than the beam size. Regions were chosen by following the features observed in the spectral index maps and with the goal of obtaining a signal-to-noise ratio higher than 3 in each region. Spectral index profiles of the E relic and of the $\mathrm{W}$ relic and the halo emission are shown in Figs 7 and 8, respectively.

\subsubsection{The eastern relic}

The integrated spectral index found in the E relic, including the NAT, is in agreement with the one obtained by George et al. (2017) with a power-law fit of the spectrum between $118 \mathrm{MHz}$ and $1.4 \mathrm{GHz}$, $\alpha_{1.4 \mathrm{GHz}}^{118 \mathrm{MHz}}=1.03 \pm 0.12$. No spectral curvature is thus observed for this relic up to $4 \mathrm{GHz}$.

Figure 6. Top panel: Spectral index image of the $\mathrm{W}$ relic and halo region computed between 1.5 and $3 \mathrm{GHz}$. Point-like sources were subtracted. The two dashed red polygons mark the flux density extraction regions for the halo and the West relic. Bottom panel: Error map of the spectral index image. Contours and restoring beam are as in Fig. 5.

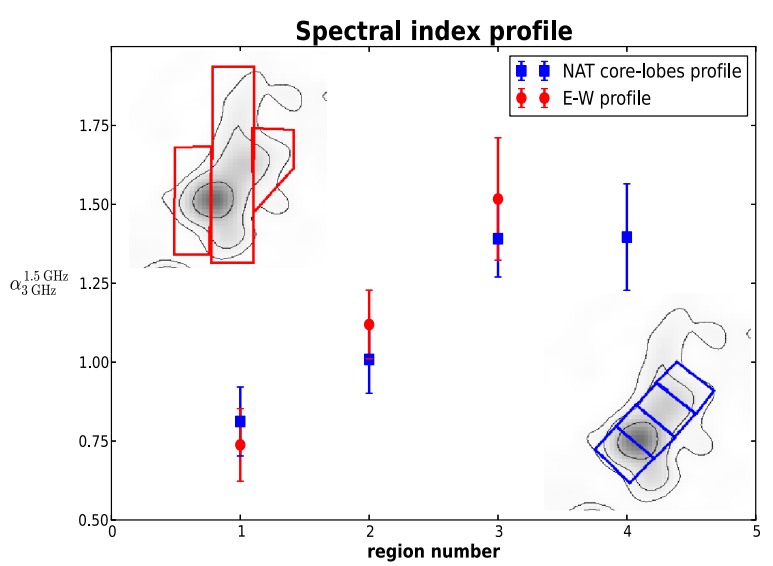

Figure 7. Spectral index profiles in the E relic. The spectral index is computed in the regions shown in the inset panels and numbered following the profiles. The regions used for the red profile (plotted with round marks) are shown in the top-left inset panel and are numbered from E to W. The regions used for the blue profile (plotted with square marks) are shown in the bottom-right inset panel and are numbered from the core in the SE to the lobes in the NW. We drew the regions in order to avoid point-like sources surrounding the extended emission. 


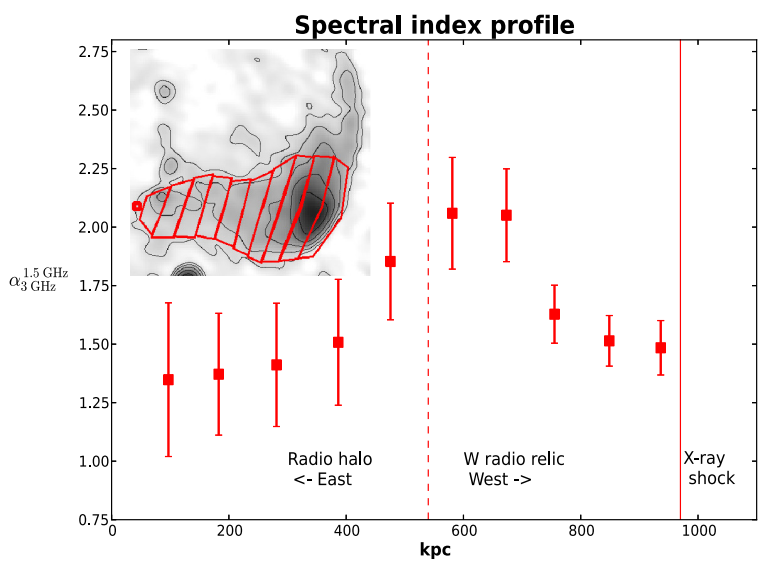

Figure 8. Spectral index profile in the $\mathrm{W}$ relic and halo region where pointlike sources were subtracted. The origin of the $x$-axis coincides with the position marked with the red point in the inset panel. The solid red line shows the position of shock front detected in X-ray while the dashed line separates the $\mathrm{W}$ relic and halo region.

The spectral index image is shown in Fig. 5. It is not trivial to identify a single spectral gradient of the whole extended emission. A steepening trend is visible from the core of the radio galaxy to its lobes but it is possible to draw a steepening trend also from $\mathrm{E}$ to $\mathrm{W}$. The first trend is expected if the only radio galaxy originates the emission while the second is expected from the acceleration of particles from a shock wave propagating outwards from $\mathrm{W}$ to $\mathrm{E}$. If these two trends were both present they would be mixed in the spectral index map, due to the physical superimposition and of the resolution of our image.

In Fig. 7, we trace both the expected trends and the spectral index profiles are displayed in different colours. The blue profile follows the core-lobes direction as can be derived from the high-resolution A configuration image at a position angle of $-45^{\circ}$ (see Fig. 1). The flattest spectral index corresponds to the core of the radio galaxy in the first blue region, with $\alpha=0.8 \pm 0.1$. The spectral index steepens towards the tails of the radio galaxy and in the fourth region, furthest away from the core, it remains constant. The fact that the northern and southern edges of the emission do not follow the core-lobes direction and show a flatter spectral index (Fig. 5) than the fourth region of the profile, indicates that the propagation of jets has been perturbed and/or that another mechanism is possibly accelerating particles.

With the red boxes we traced the E-W profile (avoiding nearby point-like sources in the $\mathrm{W}$ and $\mathrm{E}$ of the extended emission). A clear steepening trend is observed also in this direction. This is suggestive of an acceleration process that is active along the whole length of the $\mathrm{E}$ relic and not only originating in the core of the radio galaxy. This scenario is further discussed in Section 6.1.

\subsubsection{The western relic and the radio halo}

We computed the spectral index profile of the western emission region using ten regions between the centre of the cluster to the region of the shock detected in the X-ray (see Fig. 8). The emission of point-like sources was subtracted out of the images as explained in Section 4.2.

In the region close to the shock front, the spectral index is $\alpha$ $=1.5 \pm 0.1$. In Section 6.2, we derive the Mach number of the underlying shock wave assuming the DSA mechanism and compare it to the one derived from X-rays.

The spectral index steepens towards the cluster centre, reaching a peak value of $\alpha=2.1 \pm 0.2$ at $\sim 400 \mathrm{kpc}$ from the shock front. A steepening trend is expected in the downstream of a shock wave where energetic particles cool down, and it is often observed in radio relics (e.g. Hoang et al. 2017).

Towards the halo region, the spectral index flattens again. The trend is clear also from Fig. 6. The spectral profile strongly resembles the one observed in the Toothbrush radio relic (van Weeren et al. 2016). The flattening of the spectrum can be explained by the presence of another mechanism re-accelerating particles in the central region of the cluster. This is further discussed in Section 6.3.

The profile flattens and remains almost constant in the four central regions. According to the spectral index profile, we disentangled the region of the relic from that of the radio halo. We assumed that the steepening of the spectrum up to $\alpha \sim 2$ is due to the aging of particles in the region downstream the shock. We considered as radio halo the region where the spectral index flattens again. In Fig. 6, we show the two approximate regions with red dashed polygons. In Table 3, we reported average spectral index and radio power of $\mathrm{W}$ relic and radio halo.

George et al. (2017) derived a spectral index of $\alpha_{1.4 \mathrm{GHz}}^{118 \mathrm{MHz}}=1.23 \pm$ 0.09 for the western relic subtracting the flux density of the radio halo extrapolated from the $610 \mathrm{MHz}$ measurement. We instead derived two distinct integrated values: in the halo $\alpha=1.3 \pm 0.2$ and in the relic $\alpha=1.6 \pm 0.1$. We can also estimate the spectral index of the halo between $610 \mathrm{MHz}$ and $1.5 \mathrm{GHz}$ using the work of Venturi et al. (2007) and integrating over the same physical region: $\alpha_{1.5 \mathrm{GHz}}^{610 \mathrm{MHz}}=1.1 \pm 0.1$. Our spectral index estimates within the uncertainties are consistent with those measured by Venturi et al. (2007) at $610 \mathrm{MHz}$. We did not detect any signs of curvature in the integrated spectrum within the sampled frequency range.

\section{POLARIZED INTENSITY STUDY}

\subsection{Theoretical background}

Following Burn (1966) we define the linear polarization vector $P$ as a complex quantity:

$P=Q+i U=|P| e^{2 i \chi}$,

where $\chi$ is the polarization angle of the radiation and $Q$ and $U$ are the Stokes parameters.

The high degree of fractional polarization $p=P / I$ observed from radio relics unveils the presence of ordered magnetic field components lying in the plane of the sky $\left(B_{\perp}\right)$. Faraday depolarization may decrease the observed fractional polarization at large wavelength depending on the physical properties of the magneto-ionic medium between the source and the observer (Sokoloff et al. 1998).

The net component of the magnetic field along the line of sight $\left(B_{\| 1}\right)$ is responsible for the Faraday rotation on the radiation passing through the magneto-ionized ICM. The rotation of the observed polarization angle depends on $\lambda^{2}$, being $\lambda$ the observing frequency:

$\chi\left(\lambda^{2}\right)=\chi_{0}+\phi \lambda^{2}$,

where $\chi_{0}$ is the intrinsic polarization angle. The Faraday depth $\phi$ is defined as

$\phi=0.81 \int_{\text {source }}^{\text {observer }} n_{e} B_{\|} \mathrm{d} l \quad\left(\operatorname{rad~m}^{-2}\right)$, 
where $n_{\mathrm{e}}$ is the thermal electron density in $\mathrm{cm}^{-3}, B_{\|}$is the magnetic field component parallel to the line of sight in $\mu \mathrm{G}$ and $\mathrm{d} l$ is the infinitesimal path-length in parsecs.

The RM and the Faraday depth coincide at all wavelengths only when one or several (not emitting) screens lie in between the source and the observer and in the absence of beam depolarization. In this case we term the source Faraday-simple since the RM - or $\phi$ - can be recovered from equation (3).

In a general case polarized synchrotron radiation may originate in the same volume that causes Faraday rotation. In particular, in galaxy cluster we expect the magnetic field to be filamentary and the emitting volume of radio relic to be filled with turbulent thermal gas. Hence, polarized emission can be spread over a range of $\phi$ determining a Faraday-complex source.

The RM synthesis technique developed by Brentjens \& de Bruyn (2005) introduces the Faraday dispersion function, hereafter also called Faraday spectrum, $F(\phi)$, which describes the complex polarization vector as a function of the Faraday depth. The reconstructed Faraday spectrum, $\widetilde{F}(\phi)$, can be recovered by Fourier transform of the observed polarization as a function of wavelength squared. The Rotation Measure Sampling Function (RMSF) describes the instrumental response to the polarized signal in the Faraday space based on the wavelength coverage of the observation. We refer to Brentjens \& de Bruyn (2005) for details on this technique.

The amplitude of the reconstructed $\widetilde{F}(\phi)$ peaks at the Faraday depth $\phi_{\text {peak }}$, which is the Faraday depth along the path between the observer and the source contributing the most to the polarized emission. From the value of the reconstructed $\left|\widetilde{F}\left(\phi_{\text {peak }}\right)\right|$ it is possible to recover the polarization fraction of the emission, while from the reconstructed Stokes parameters, $\widetilde{Q}\left(\phi_{\text {peak }}\right)$ and $\widetilde{U}\left(\phi_{\text {peak }}\right)$, we can recover the polarization angle at $\lambda_{0}^{2}$ (i.e. the weighted average of the observed bandwidth):

$\chi\left(\lambda_{0}^{2}\right)=\frac{1}{2} \arctan \frac{\widetilde{U}\left(\phi_{\text {peak }}\right)}{\widetilde{Q}\left(\phi_{\text {peak }}\right)}$.

\subsection{Polarized intensity imaging}

We made use of WSCLEAN 2.6 (Offringa et al. 2014) for the polarization intensity imaging. This imager exploits a $w$-stacking algorithm as a faster alternative to $w$-projection and allows multiscale, multifrequency, and automasking algorithms (Offringa \& Smirnov 2017).

We imaged 1-2 GHz A, B, C configurations data and 2-4 GHz data in DnC configuration to sample different spatial scales and frequencies. We also used together $L$-band $\mathrm{B}+\mathrm{C}$ and $S$-band $\mathrm{DnC}$ data to cover the whole frequency band $1-4 \mathrm{GHz}$. The images were cleaned down to $3 \sigma$ level using the automasking option. We used 64 frequency sub-bands of $16 \mathrm{MHz}$ each for the data in $L$ band, and 16 sub-bands of $128 \mathrm{MHz}$ for the $S$-band data. The different sub-band width is required to avoid bandwidth depolarization at the lowest frequency. For the whole $1-4 \mathrm{GHz}$ band, we used 96 frequency sub-bands of $32 \mathrm{MHz}$ each. The restoring beam was forced to be the same in each frequency sub-band, matching the lowest resolution one. The parameters used for each image are listed in Table 4.

We used join-channels and join-polarizations options to make Stokes $I, Q, U$ image cubes and full-bandwidth images, as recommended in the WSCLEAN documentation. Each image was corrected for the primary beam calculated for the central frequency of the sub-band. We restricted our analysis to a circular region of radius $\sim 6$ arcmin (i.e. $\sim 1.4 \mathrm{Mpc}$ ) around the $L$ band pointing centre so that the effect of direction-dependent gain, polarization leakage in $Q$ and $U$, and beam squint are negligible (Jagannathan et al. 2017). We quantified the leakage from Stokes $I$ to $V$ to be $\leq 2$ per cent within the closest 6 arcmin to the image centre. This constrains the leakage to Stokes $Q$ and $U$ to be within 1 per cent of I. We caution however about the usage of similar data for sources showing lower fractional polarization ( $<5$ per cent) and further away from the beam centre.

\subsection{RM synthesis}

We performed RM synthesis on the $Q$ and $U$ image cubes using PYRMSYNTH ${ }^{6}$ and we obtained the cubes in the Faraday space. We thus recovered the reconstructed Faraday dispersion function, or Faraday spectrum, $\widetilde{F}(\phi)$, in each pixel (i.e. each line of sight).

Faraday cubes were created between $\pm 600 \mathrm{rad} \mathrm{m}^{-2}$ and using bins of $2 \mathrm{rad} \mathrm{m}^{-2}$. This range is motivated by our sensitivity to large values of $\phi$. From Brentjens \& de Bruyn (2005) we can estimate the resolution in Faraday space (i.e. the FWHM of the main peak of the RMSF), $\delta \phi$, the maximum observable Faraday depth, $\left|\phi_{\max }\right|$, and the largest observable scale in Faraday space, $\Delta \phi_{\max }$ (i.e. the depth and the $\phi$-scale at which sensitivity has dropped to 50 per cent). The parameters for each observation are listed in Table 4.

Notice the different resolutions and $\Delta \phi_{\max }$ values of our measurements. The values of $\left|\phi_{\max }\right|$ to which we are sensitive are well above the value expected from galaxy clusters (see e.g. Bonafede et al. 2010, 2013; Böhringer, Chon \& Kronberg 2016). In this galaxy cluster, we observed $\left|\phi_{\text {peak }}\right|<100 \mathrm{rad} \mathrm{m}^{-2}$ both in the $L$ and in the $S$-band observations, so that the lower $\left|\phi_{\max }\right|$ obtained combining $L$ - and $S$ band does not limit our measurements. In the combined data set, with a central frequency of $2.5 \mathrm{GHz}$, we reach the highest resolution in Faraday space and we are sensitive to polarized emission spread over large Faraday scales.

We first run PYRMSYNTH on the entire central region cleaning the spectrum down to five times the noise level of full-bandwidth $Q$ and $U$ images (see Heald 2009, for the RM clean technique). We imposed an average total intensity spectral index $\alpha=1$ on the entire field. We noticed the RM cleaning process was improved by the use of $\alpha=1$ instead of the default $\alpha=0$, although it comes from an average estimate for the entire field and it is assumed to be constant at each Faraday depth. We measured the local rms noises $\sigma_{Q}$ and $\sigma_{U}$ in the slices of $\widetilde{Q}(\phi)$ and $\widetilde{U}(\phi)$ at $500 \mathrm{rad} \mathrm{m}^{-2}<|\phi|<600 \mathrm{rad} \mathrm{m}^{-2}$ i.e. outside the sensitivity range of our observations. Since $\sigma_{Q} \sim \sigma_{U}$, we estimated the noise of polarization observation as $\sigma_{Q U}=\left(\sigma_{Q}+\sigma_{U}\right) / 2$ (see also Hales et al. 2012). This value is reported in Table 4 for each measurement set. Then, we selected pixels with a peak in the Faraday spectrum above a threshold of $8 \sigma_{Q U}$, following George, Stil \& Keller (2012), which corresponds to a false detection rate of 0.06 per cent and to a Gaussian significance level of about $7 \sigma$ according to Hales et al. (2012). This conservative choice accounts for the Ricean bias (i.e. the overestimation of polarized intensity due to $P$ being positivedefinite and governed by the Ricean distribution), the non-Gaussian noise in the $Q$ and $U$ images, and the additional bias due to error in $\phi_{\text {peak }}$ estimates. We run again pyrmsynth only on these pixels, cleaning the spectrum down to $8 \sigma_{Q U}$ level. 
Table 4. Details of polarized intensity images. Column 1: observing frequency range, i.e. first and last frequency sub-bands; Column 2: name of the frequency band; Column 3: array configuration; Column 4: $\delta v$ is the frequency sub-band width; Column 5: FWHM of the major and minor axes of the restoring beam of the image cubes; Column 6: $\sigma_{Q U}$ is the best estimate for the rms noise in polarization obtained as $\left(\sigma_{Q}+\sigma_{U}\right) / 2$; Column 7: resolution in the Faraday space; Column 8: maximum observable Faraday depth; Column 9: largest observable Faraday scale; Column 10: reference of the figures in this paper.

\begin{tabular}{|c|c|c|c|c|c|c|c|c|c|}
\hline $\begin{array}{l}\text { Freq. range } \\
(\mathrm{GHz})\end{array}$ & Band & Array config. & $\begin{array}{c}\delta v \\
(\mathrm{MHz})\end{array}$ & Beam & $\begin{array}{c}\sigma_{Q U} \\
\left(\mathrm{mJy} \text { beam }^{-1}\right)\end{array}$ & $\begin{array}{c}\delta \phi \\
\left(\operatorname{rad~m^{-2}}\right)\end{array}$ & $\begin{array}{c}\left|\phi_{\max }\right| \\
\left(\operatorname{rad~m}^{-2}\right)\end{array}$ & $\begin{array}{c}\Delta \phi_{\max } \\
\left(\operatorname{rad~m^{-2}}\right)\end{array}$ & Figure \\
\hline $1.015-2.023$ & $L$ & A & 16 & $\begin{array}{c}2.5 \operatorname{arcsec} \times \\
2.5 \operatorname{arcsec}\end{array}$ & 0.004 & 45 & 535 & 143 & \\
\hline $1.015-2.023$ & $L$ & B & 16 & $\begin{array}{c}11 \operatorname{arcsec} \times \\
5 \operatorname{arcsec}\end{array}$ & 0.005 & 45 & 535 & 143 & \\
\hline $1.015-2.023$ & $L$ & $\mathrm{C}$ & 16 & $\begin{array}{c}25 \operatorname{arcsec} \times \\
25 \operatorname{arcsec}\end{array}$ & 0.007 & 45 & 535 & 143 & 9,10 \\
\hline $1.022-3.995$ & $S+L$ & $\mathrm{~B}+\mathrm{C}+\mathrm{DnC}$ & 32 & $\begin{array}{c}25 \operatorname{arcsec} \times \\
25 \operatorname{arcsec}\end{array}$ & 0.004 & 37 & 288 & 558 & 11,12 \\
\hline
\end{tabular}

We computed polarization intensity images using the peak of the Faraday dispersion function and correcting for the Ricean bias as $P=\sqrt{\left|\widetilde{F}\left(\phi_{\text {peak }}\right)\right|^{2}-2.3 \sigma_{Q U}^{2}}$ (George et al. 2012). We then obtained fractional polarization images dividing the $P$ images (with the $8 \sigma_{Q U}$ threshold) by the full-band Stokes $I$ images with a cut-off of three times the rms noise. We also obtained $\phi_{\text {peak }}$ images with the same cut-off. From the reconstructed values of $Q$ and $U$ at $\phi_{\text {peak }}$ we can also recover the intrinsic polarization angle (i.e. corrected for the value of RM determined by $\left.\phi_{\text {peak }}\right), \chi_{0}$, as

$\chi_{0}=\chi\left(\lambda_{0}^{2}\right)-\phi_{\text {peak }} \lambda_{0}^{2}=\frac{1}{2} \arctan \frac{\widetilde{U}\left(\phi_{\text {peak }}\right)}{\widetilde{Q}\left(\phi_{\text {peak }}\right)}-\phi_{\text {peak }} \lambda_{0}^{2}$,

where $\lambda_{0}$ is $19.7 \mathrm{~cm}, 11.9 \mathrm{~cm}$ and $10 \mathrm{~cm}$ for the $L, S+L$, and $S$ band, respectively.

Fractional polarization and magnetic field vector images of the $L$-band $\mathrm{C}$ configuration and $S$ band are shown in Figs 9 and 10 for the $\mathrm{E}$ and $\mathrm{W}$ relics, respectively. We also obtained $\mathrm{B}$ configuration $L$-band images but, for the purpose of this work, we are more interested in the polarized diffuse emission of the relics, more visible in the $\mathrm{C}$ configuration image. We obtained only few pixels above the $8 \sigma_{Q U}$ cutoff with the A configuration $L$-band image. Fractional polarization and magnetic field vector images obtained from the whole frequency band $1-4 \mathrm{GHz}$ are shown in the upper panel of Fig. 11. We show the same quantities obtained at different frequency bands to show the importance of combining multiband observations for this analysis.

The Faraday depth image of RXC J1314.4-2515 resulting from the combined $S+L$-band data set is shown in Fig. 12 . We show only the $S+L$-band map since we obtained a good trade-off between resolution in Faraday space and sensitivity. Thanks to the wide and contiguous frequency coverage of our observations, we can identify some regions of the $\mathrm{W}$ relic that clearly show Faraday-complex structures. In this case, the value of $\left|\widetilde{F}\left(\phi_{\text {peak }}\right)\right|$ and of $\phi_{\text {peak }}$ are not sufficient to describe the polarization and rotation effect experienced by the radiation since the polarized emission would be spread at different values of $\phi$ and affected by Faraday depolarization. For this reason, the results of RM-synthesis in the southern region of the $\mathrm{W}$ relic (i.e. the nose) should be regarded with caution. Faradaycomplex structures are separately discussed in Section 6.6.

The RM value of the Galactic foreground (i.e. $-30 \mathrm{rad} \mathrm{m}^{-2}$ ) was subtracted out: we will refer to the cluster Faraday depth, $\phi_{\mathrm{cl}}$, to indicate foreground subtracted values.

\subsubsection{Uncertainties on the measure of $\phi$}

The method commonly used to estimate the uncertainties on $\phi$ is derived from Brentjens \& de Bruyn (2005) where

$\sigma_{\phi}=\frac{\delta \phi}{2 P / \sigma_{Q U}}$

that is the half width at half-maximum of the RMSF divided by the signal to noise of the detection. This expression is derived under the assumption $\alpha=0$ and $\sigma_{Q}=\sigma_{U}$. In other cases, it can lead to over- or underestimates of the errors (Schnitzeler \& Lee 2017). However, we computed $\sigma_{\phi}$ since it can be useful to compare the uncertainties pixel by pixel, and we added in quadrature the error of $2 \mathrm{rad} \mathrm{m}^{-2}$ on the estimate of the Galactic foreground by Taylor et al. (2009) to obtain $\sigma_{\phi_{\mathrm{cl}}}$. The error map is shown in the bottom panel of Fig. 12. From $\sigma_{\phi}$ we derived the error on the polarization angle by propagating the uncertainties on the quantities in equation (6) as

$\sigma_{\chi_{0}}^{2}=\sigma_{\chi}^{2}+\sigma_{\phi}^{2} \lambda_{0}^{4}=\frac{\sigma_{Q U}^{2}}{4 P^{2}}+\left(\frac{\delta \phi}{2 P / \sigma_{Q U}}\right)^{2} \lambda_{0}^{4}$.

These uncertainties for the vector map for the $S+L$-band data set are shown in the bottom panel of Fig. 11.

As pixel values are not independent of each other, in the text we will refer to beam-averaged quantities, which are the average values of $\phi_{\mathrm{cl}}$ over beam-size regions, weighted by the signal-tonoise ratio of pixels. To a first approximation, in the case of Faraday-simple sources, the distribution of RM values in each beam has a Gaussian distribution for signal-to-noise ratios higher than 8 (George et al. 2012) and its variance is not due to a physical variation of the Faraday rotating medium, but to the underling noise in the Faraday spectrum, which shifts the position of the peak. Hence, we calculated also the standard deviation of neighbouring pixels in a region equivalent to the beam area to estimate the RM uncertainties.

\subsubsection{The eastern relic}

In the B configuration, we detected polarized emission arising only from a barely resolved source in the south-east of the E relic (source $\mathrm{C}$ in Fig. 1) and from two regions close to the lobes of the NAT radio galaxy embedded in the relic.

In the $S$-band and $L$-band C configuration images (Fig. 9), the average polarization fraction of the source $C$ is $4.3 \pm 0.4$ per cent and $3.6 \pm 0.4$ per cent, respectively. The polarization fraction is 

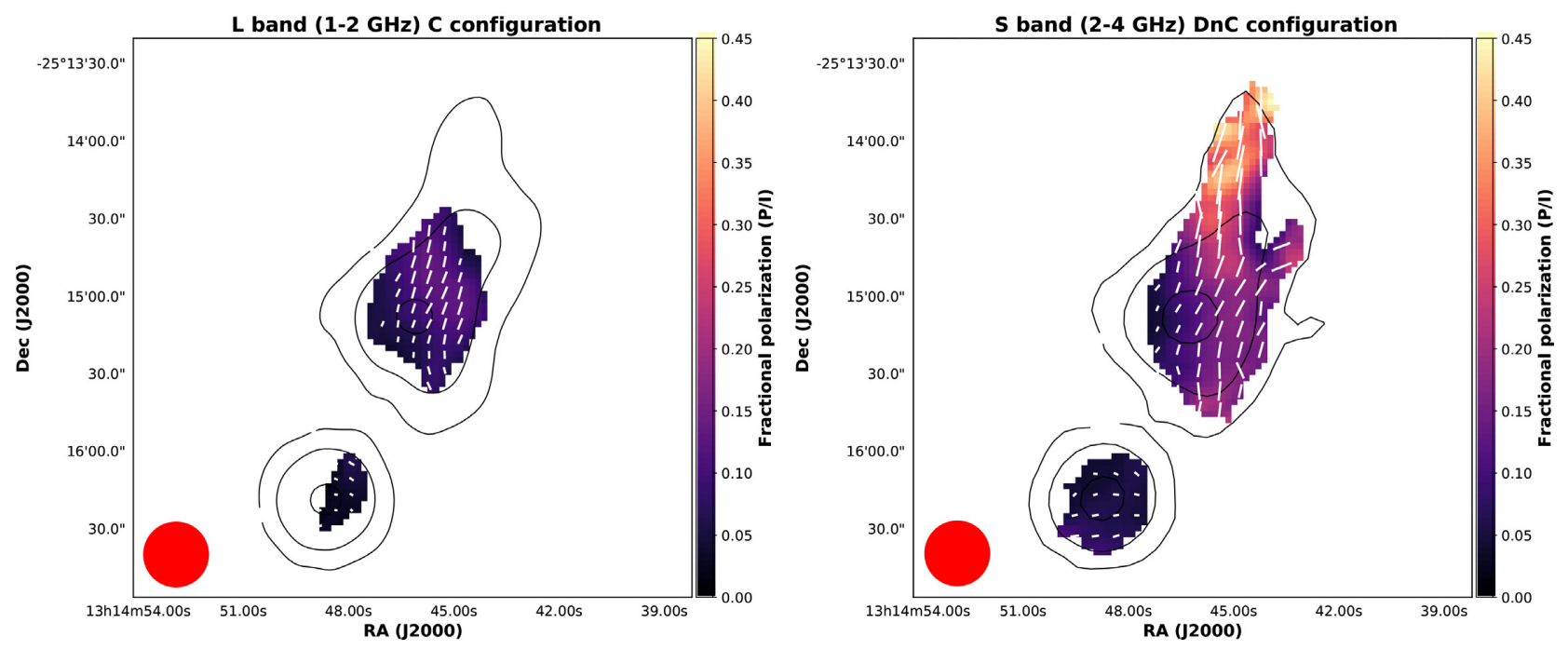

Figure 9. Fractional polarization (colour scale) and magnetic field vectors (white lines) of the $\mathrm{E}$ radio relic: $L$-band data in $\mathrm{C}$ configuration (left-hand panel) and $S$-band data (right-hand panel). An $8 \sigma Q U$ detection threshold was imposed in polarization and values were corrected for the Ricean bias. See Table 4 for details on the images. The length of white vectors is proportional to the fractional polarization. Black contours are from the total intensity images in the same frequency band and configuration. The restoring beam is $25 \operatorname{arcsec} \times 25 \operatorname{arcsec}$ in both images. Contour levels start from three times the rms noise $(0.08$ and $0.03 \mathrm{mJy}$ beam $^{-1}$ for the $L$ band and $S$ band, respectively) and are spaced by a factor of 4 .
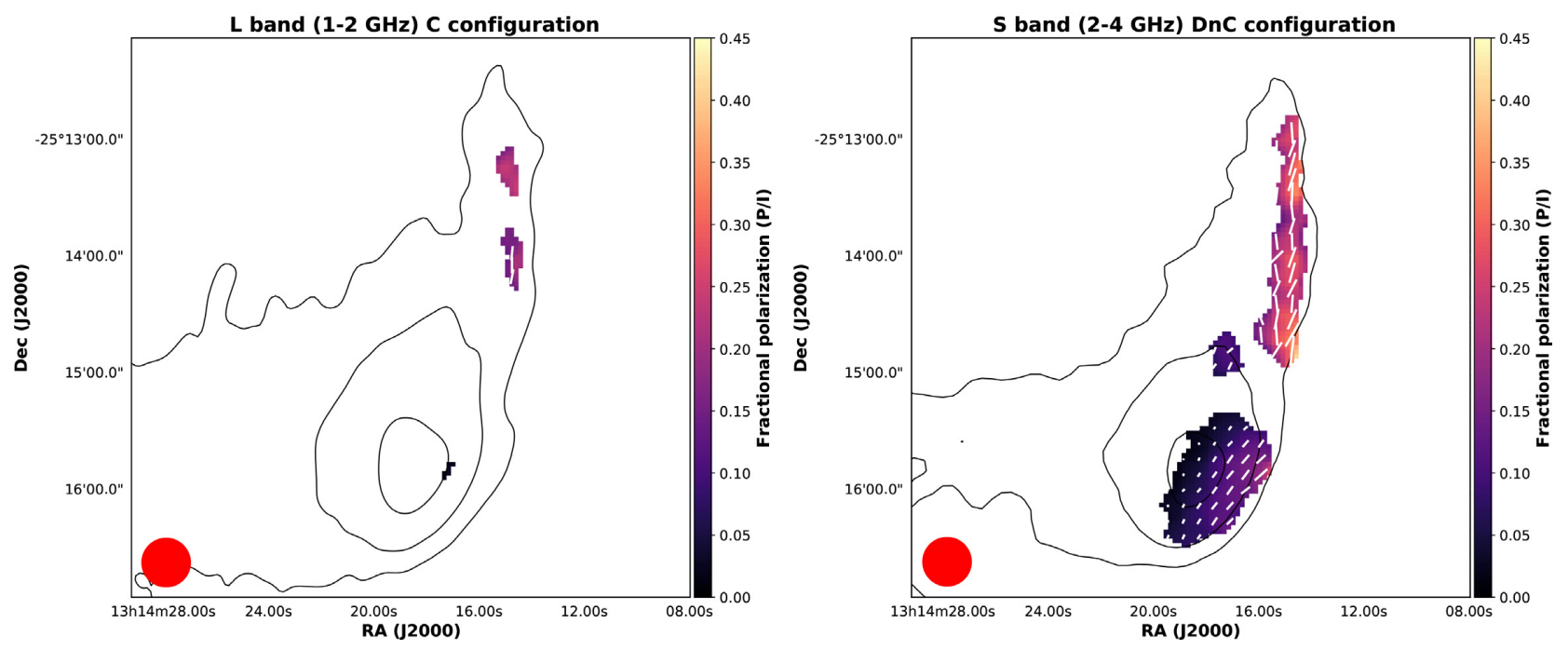

Figure 10. Same as Fig. 9 for the W relic.

3.6 \pm 0.3 per cent also in the combined $S+L$-band image (Fig. 11). The weighted average $\phi_{\mathrm{cl}}$ of this source derived from the Faraday depth map of Fig. 12 is $-4 \mathrm{rad} \mathrm{m}^{-2}$ while the standard deviation is $9 \mathrm{rad} \mathrm{m}^{-2}$.

The $\mathrm{E}$ relic shows a resolved polarized emission in the $\mathrm{C}$ configuration $L$-band and in the $S$-band images (Fig. 9). The fractional polarization observed in the $L$ band is lower than what is detected in other radio relics, while in the $S$ band it is consistent with the literature (van Weeren et al. 2019). The region of the core of the NAT radio galaxy in the eastern edge of the relic is polarized at an average $9.9 \pm 0.9$ per cent level in the $S$ band. The fractional polarization increases in the region of the lobes and in the $\mathrm{N}-\mathrm{S}$ direction. In particular, in the $S$-band image we detect polarized emission from the $\mathrm{E}$ radio relic, reaching a polarization fraction of $\sim 45$ per cent in the northern part of the relic. The magnetic field is oriented almost in the direction of the radio lobes of the galaxy and then bends to be aligned in the $\mathrm{N}-\mathrm{S}$ direction along the radio relic. The same is observed in Fig. 11.

The values of average $\phi_{\mathrm{cl}}$ within beam-size regions located in the $\mathrm{E}$ relic are almost constant ranging between 2 and $4 \mathrm{rad} \mathrm{m}^{-2}$ with a standard deviation of $\sim 3 \mathrm{rad} \mathrm{m}^{-2}$. Only in the northern side of the relic the average $\phi_{c l}$ changes to $-3 \mathrm{rad} \mathrm{m}^{-2}$ with a standard deviation of $11 \mathrm{rad} \mathrm{m}^{-2}$.

All the values of $\phi_{\mathrm{cl}}$ measured are consistent with zero considering the standard deviation as the uncertainty, meaning that the electron density and the magnetic field in this region of the cluster are not responsible for the Faraday rotation effect that is mainly due to the external screen of our Galaxy. This is confirmed by the moderate Faraday depolarization detected in this region that can be explained by the low thermal electron density (see Fig. 2). 

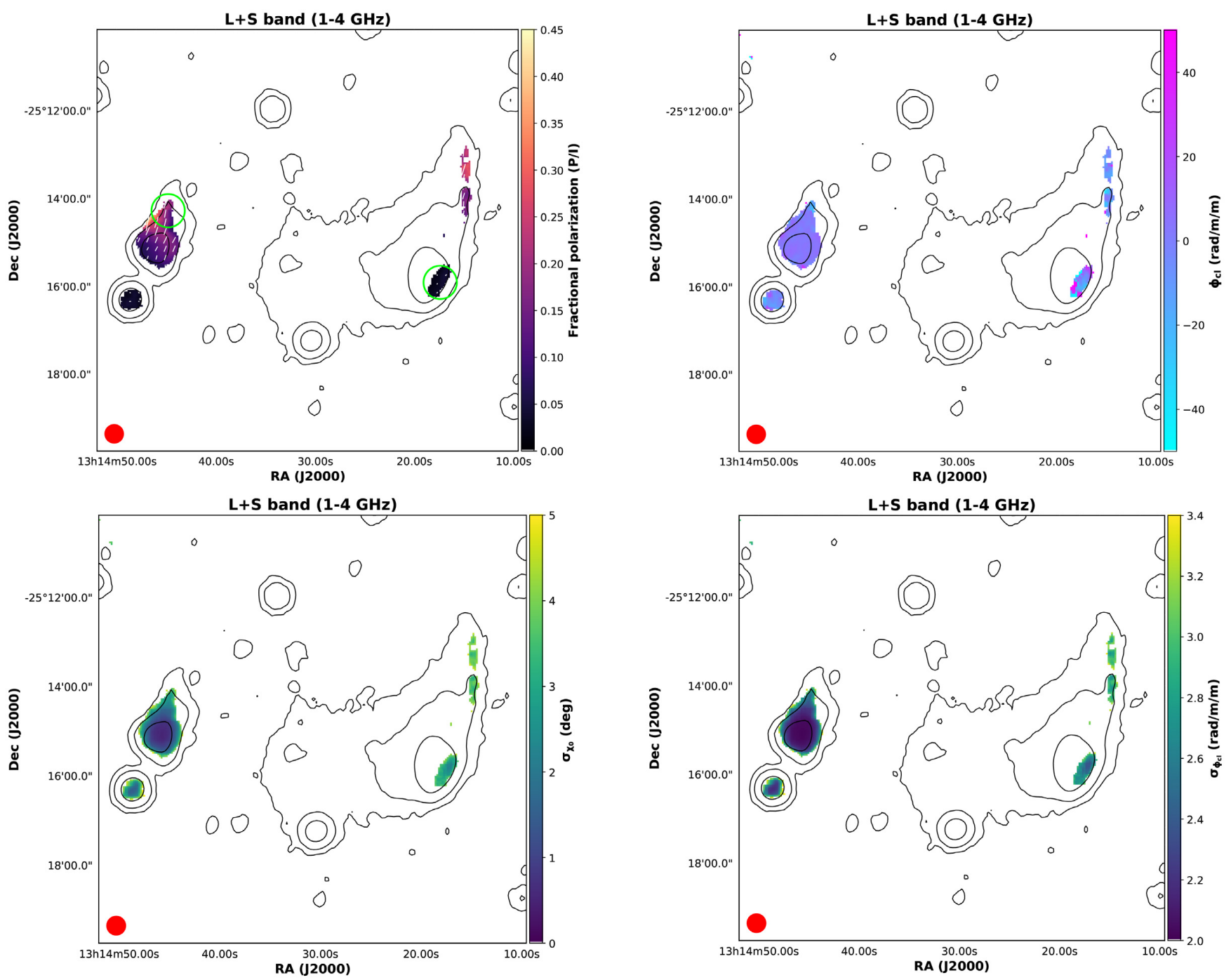

Figure 11. Top panel: Fractional polarization (colour scale) and magnetic field (white lines) with a $8 \sigma_{Q U}$ detection threshold for the $1-4 \mathrm{GHz}$ data in $\mathrm{B}+\mathrm{C}+\mathrm{DnC}$ configuration (see Table 4 for image details). Fractional polarization values were corrected for the Ricean bias. The green circles mark the regions used for the Faraday depolarization study in Section 6.6. The length of white vectors is proportional to the fractional polarization. Bottom panel: Error map of the polarization angle. In both panels, the black contours are from the total intensity image with a restoring beam of 25 arcsec $\times 25$ arcsec, shown in the left-hand corner of the image. They start from three times the rms noise of $0.02 \mathrm{mJy}^{\text {beam }}{ }^{-1}$ and are spaced by a factor of 4.

\subsubsection{The western relic and the radio halo}

The western side of RXC J1314.4-2515 does not show polarization in the B configuration image. Few pixels with a signal-to-noise ratio in polarization higher than 8 appear in the thinnest part of the outer $\mathrm{W}$ relic in the $L$-band $\mathrm{C}$ configuration image. Here, the degree of polarization reaches the 25 per cent level but it is measured only in few pixels (Fig. 10). Hence, the fractional polarization observed in the $L$ band is lower than what expected from the literature also for the W relic (see van Weeren et al. 2019, and references therein).

In the $S$ band, we found a totally different situation (see Fig. 10). Almost all of the outer arc shows high levels of intrinsic fractional polarization reaching a value of $\sim 40$ per cent in the northern part. The average fractional polarization in the northern arc is

Figure 12. Top panel: Value of $\phi_{\mathrm{cl}}$ on a pixel by pixel basis with a $8 \sigma_{Q U}$ detection threshold for the $1-4 \mathrm{GHz}$ image in $\mathrm{B}+\mathrm{C}+\mathrm{DnC}$ configuration. Bottom panel: Error map of the $\phi_{\mathrm{cl}}$ image. Black contours and restoring beam are the same as Fig. 11.

$24 \pm 4$ per cent. The magnetic field lines are almost aligned along the radio relic arc. In Fig. 12, the values of $\phi_{\mathrm{cl}}$ in the northern part of the relic are scattered between -44 and $42 \mathrm{rad} \mathrm{m}^{-2}$. A spot of polarized emission, without any optical counterpart, is also detected along the inner arc at $10 \pm 3$ per cent level of polarization and with values of $\phi_{\mathrm{cl}}$ reaching $50 \mathrm{rad} \mathrm{m}^{-2}$.

Also the southern part of the arc (i.e. the nose) shows polarization with an average value of $7.6 \pm 0.6$ per cent in the $S$-band and of $3.0 \pm 0.3$ per cent in the $S+L$ combined data set. In the $S$ band, the fractional polarization increases, from $\sim 2$ per cent in the inner region to $\sim 25$ per cent at the shock front detected in the X-rays (see right-hand panel of Fig. 10). In the whole southern region, the $\phi_{\mathrm{cl}}$ values in the $S+L$ band are in the range between -82 and 78

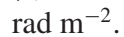

The emission coincident with the shock region, detected only in the $S$ - and $S+L$-band observations, clearly shows a Faradaycomplex emission unveiled by a broadening of the Faraday spectrum larger than the FWHM of the RMSF (i.e. at least larger than 150 $\mathrm{rad} \mathrm{m}^{-2}$, see Fig. 13). The strong Faraday depolarization between $S$ - and $L$ band in this region is probably explained by the higher 

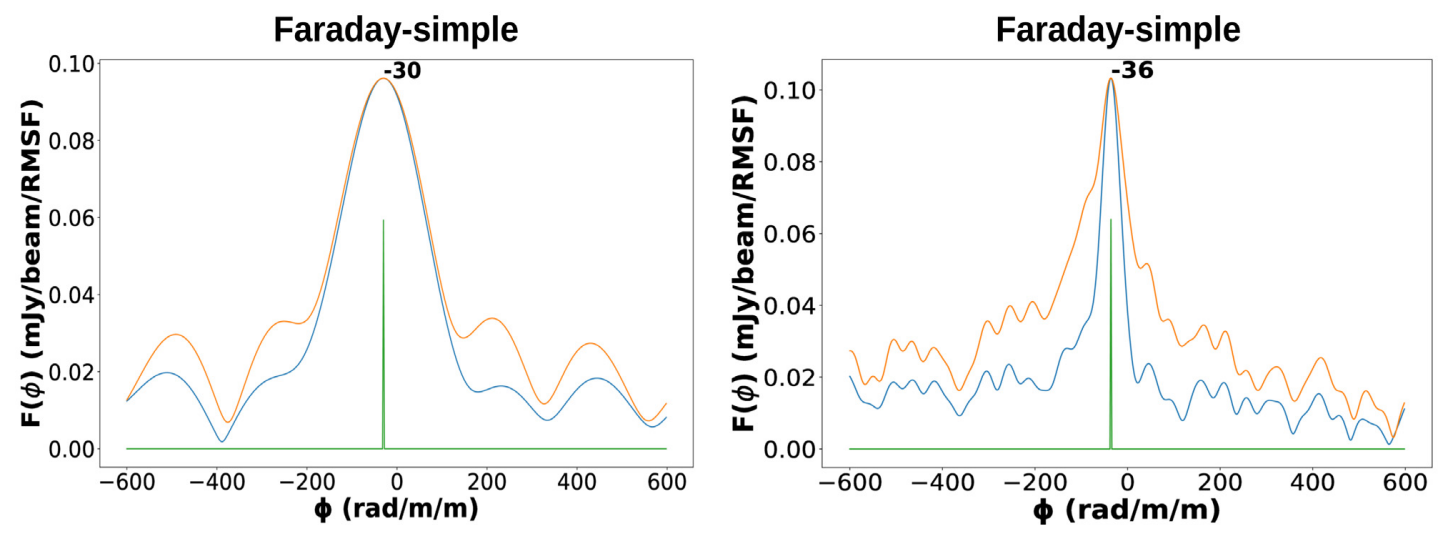

Faraday-complex
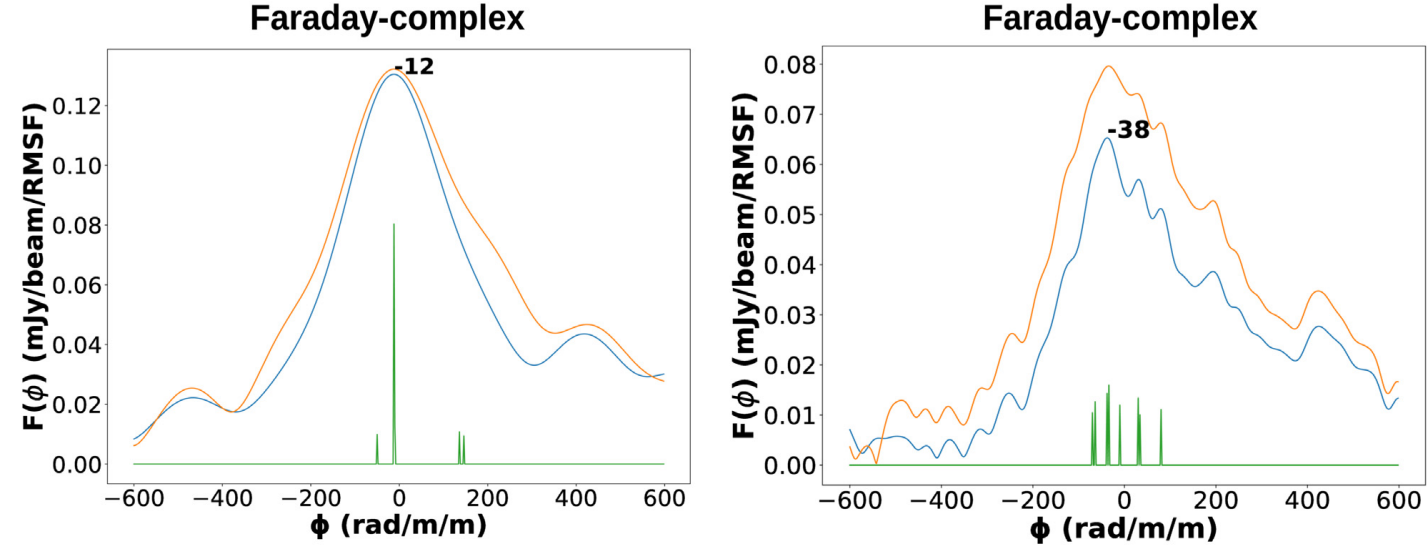

Figure 13. Faraday dispersion function of two representative pixels in the $2-4 \mathrm{GHz}$ band observation (left-hand column) and in the whole $1-4 \mathrm{GHz}$ band (right-hand column). The orange line is the dirty spectrum, the blue is the clean spectrum, and in green the clean components. The pixel in the first row has a Faraday-simple structure while the one in the second row has a Faraday-complex structure, barely resolved in the left-hand panel and resolved as a convolution of peaks in the righ-hand one.

thermal electron density in respect to the E relic (Kierdorf et al. 2017). This is further discussed in Section 6.6.

The emission that extends from the $\mathrm{W}$ relic towards the central region of RXC J1314.4-2515 does not show any polarized emission. The upper limit on the fractional polarization in this area is 17 per cent, in agreement with literature results for other radio haloes (e.g. Feretti et al. 2012).

\section{DISCUSSION}

\subsection{AGN-relic connection in the eastern relic}

The NAT radio galaxy in Fig. 1 is a cluster member, and its lobes extend for $\sim 90 \mathrm{kpc}$ fading into the radio relic that extends in the $\mathrm{N}-\mathrm{S}$ direction. Although the detection of a shock related to the $\mathrm{E}$ relic emission is prevented by the low X-ray surface brightness (see Fig. 2) the combined information gained from the spectral index and the polarized intensity study support the idea that a shock wave is at the origin of the extended eastern emission together with the AGN activity. Since pairs of shock waves propagating along the merger axis are generated during cluster mergers, a shock wave moving outwards from $\mathrm{W}$ to $\mathrm{E}$ is in fact expected to be present at the position of the relic.

Assuming a magnetic field between 0.1 and $10 \mu \mathrm{G}$, as observed in other galaxy clusters (e.g. Govoni \& Feretti 2004), the lifetime of relativistic electrons that emit at $3 \mathrm{GHz}$ at redshift 0.247 due to synchrotron and inverse Compton losses is $\leq 10^{8} \mathrm{yr}$ (van Weeren et al. 2019, equation 3). Considering a plasma diffusion and/or bulk velocity of $\sim 10^{5} \mathrm{~m} \mathrm{~s}^{-1}$ (either due to AGN jet activity or to the merger shock) these high-energy electrons are already aged after distances of few tens kpc. The observed emission at $3 \mathrm{GHz}$ spreads over $500 \mathrm{kpc}$ and implies that another mechanism is actively energizing the electrons. We suggest that the re-acceleration originates in the shock front as suggested by the E-W spectral steepening (see Section 4.2.1).

Low-energy electrons with Lorentz factor $\gamma<10^{2}$ have radiative lifetimes larger than the Hubble time. During a maximum AGN lifetime of $\sim 1$ Gyr these electrons can travel distances of hundreds $\mathrm{kpc}$ in the ICM. Hence, it is plausible that the AGN may have supplied the low-energy electrons re-accelerated by the shock.

In the northern region of the $\mathrm{E}$ radio relic, we measured an integrated spectral index of $\alpha=1.2 \pm 0.2$, flatter than the value reached in the region of the lobes of the radio galaxy (see Fig. 7). The flattening of the spectral index at the edge of the E relic could indicate that the particles are first injected by AGN jets in the ICM, where they lose energy due to synchrotron and Inverse Compton losses in the radio galaxy lobes, before being re-accelerated by a shock wave. A similar scenario was invoked by Bonafede et al. (2014) for PLCKG287.0+32.9. Recently, van Weeren et al. (2017a) reported the clearest connection known to date between an AGN and a relic in Abell 3411-Abell 3412.

If spectral index of the pre-existing population is flatter than the one that could be produced by the shock, the spectrum is amplified and retains the spectral index of the pre-existing population (e.g. 
Gabici \& Blasi 2003; Kang \& Ryu 2011). In the opposite case, the post-shock electron population has the spectral index of the DSA and loses the memory of the injection spectrum. Since we observe a flatter spectrum in the northern edge compared to the lobes region, we are in this latter case. The sampled regions have sizes $>50 \mathrm{kpc}$, thus the electron population should have already reached the equilibrium with the energy losses (continuous injection model, Kardashev 1962). Hence, the Mach of the shock can be derived from the integrated spectral index in the region (see e.g. Trasatti et al. 2015) as

$M^{2}=\frac{\alpha+1}{\alpha-1}$.

This implies that, if a shock wave is re-vitalizing the aged particles from the AGN lobes, its Mach number would be $M=3 \pm 1$.

Furthermore, polarization vectors suggest that the projected magnetic field lines follow the AGN jets and then are bent along the north-south direction (see 10). A shock wave propagating from $\mathrm{W}$ to $\mathrm{E}$ along the merger axis (i.e. the line connecting the two subclusters) would be able to align the magnetic field lines in the $\mathrm{N}-\mathrm{S}$ direction. A shock wave with $M \sim 3$ can in fact amplify the magnetic field components parallel to the shock front of a factor 2.5 (Iapichino \& Brüggen 2012), so that the resulting magnetic field on the plane of the sky would have a preferential direction aligned with the $\mathrm{N}-\mathrm{S}$ shock front. As a result, the polarization fraction of the emission is enhanced and reaches values of $\sim 50$ per cent. The fact that the highest polarization is observed in the same northern region where we found a flattening of the spectrum, suggests that in this region the shock wave could be the active acceleration process. Where the emission of the radio galaxy dominates, the fractional polarization is lower, as expected for radio galaxies. This further indicates that the plasma could be a mixture of radio-emitting particles accelerated by the AGN jets and of plasma tracing a shock wave with highly ordered magnetic fields.

The Faraday depth values of the cluster that we derived from the NAT and the radio relic (i.e. $\phi_{\mathrm{cl}}$ in Fig. 12) are in agreement and consistent with zero within the uncertainties. This indicates that in both the sources the Faraday rotation is only caused by the external screen of our Galaxy and thus, in the regions of the cluster where they lie, the ICM has similar properties (i.e. either low thermal electron density and/or low magnetic field).

We can obtain an equipartition estimate of the magnetic field in the region of the E relic. We refer to Govoni \& Feretti (e.g. 2004) for the details of this method. We integrated the synchrotron luminosity between $10 \mathrm{MHz}$ and $10 \mathrm{GHz}$. Assuming that all the relic volume is occupied by magnetic fields and have a width of $500 \mathrm{kpc}$ along the line of sight, the equipartition magnetic field is estimated to range from 0.9 to $2.7 \mu \mathrm{G}$, depending on the $k$ value for the ratio between the energy density of relativistic protons and electrons. The upper bound comes from the assumption of $k=10^{2}$, as is usually inferred for the Milky Way (Schlickeiser et al. 2002), while the lower bound is for $k=1$. A similar electron to proton ratio is implied by our modelling of the W relic, at variance with standard DSA model (see Section 6.5). This magnetic field value should be used with caution because it is based on some working and simplified assumptions. Considering our uncertainties on the RM determination, an ordered magnetic field with this strength along the line of sight would have been detected if the electron density was higher than $10^{-5} \mathrm{~cm}^{-3}$.

Overall, our analysis of the spectral index and polarization properties of the NAT and E relic supports the idea that remnants of radio lobes from AGNs are strongly related to the origin of radio relics providing a fossil, low-energy (i.e. $\gamma<10^{2}$ ) electron

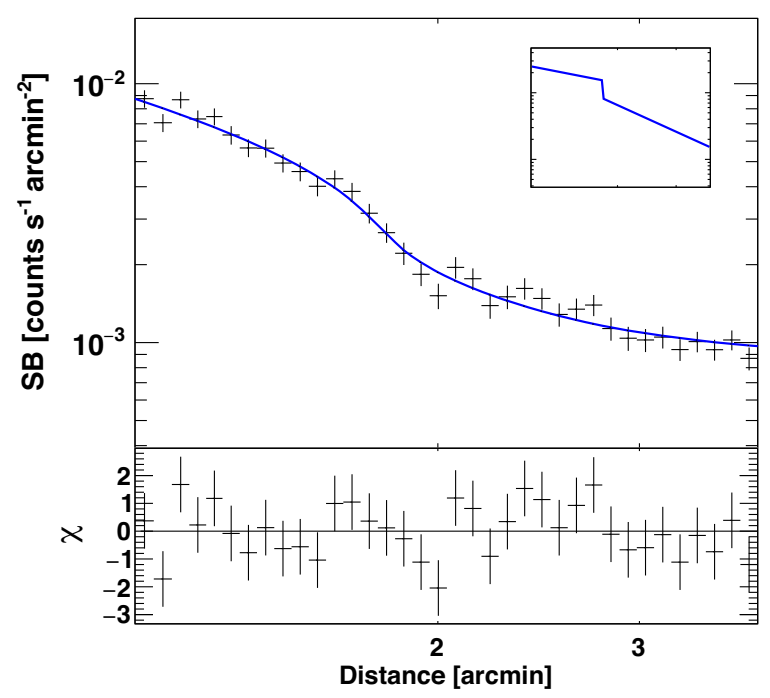

Figure 14. XMM-Newton surface brightness profile in the $0.5-2.0 \mathrm{keV}$ band extracted in the yellow sector shown in Fig. 2. The best-fitting broken power-law model provides a good description of the data $\left(\chi^{2}=32\right.$ for 31 degrees of freedom) and the discontinuity, located at a radial distance of $1.81_{-0.05}^{+0.06}$ arcmin from the centre of the sector, is coincident with the outer edge of the radio emission. The profile is convolved with the XMM-Newton point spread function. The inset panel shows the corresponding 3D gas density model.

population (Markevitch et al. 2005; Bonafede et al. 2014; Kang \& Ryu 2016; van Weeren et al. 2017a).

\subsection{Relic-shock connection in the western relic}

Our deep JVLA images show intriguing features in the western radio relic that appears constituted by two arcs in Fig. 2. A peculiar morphology is also observed in the X-rays, where a shock front with an unusual 'M' shape was reported (Mazzotta et al. 2011). We reanalysed the archival XMM-Newton observations and used our new radio images to study the connection between the shock and the radio relic.

We used PROFFIT v1.5 (Eckert, Molendi \& Paltani 2011) to extract a number of surface brightness profiles following the ' $M$ ' shape of the shock. We then decided to focus our analysis in the yellow sector reported in Fig. 2 that better highlights the discontinuity and encloses the brightest part of the radio relic. We adopted a broken power-law model to fit the data in such a region, as this model shape is generally used to describe density discontinuities in the ICM such as shocks and cold fronts (e.g. Markevitch \& Vikhlinin 2007). The best-fitting model convolved for the XMM-Newton point spread function (see Eckert et al. 2016b, for details) is reported in Fig. 14 and appears in good agreement with the data. The surface brightness jump is observed to be co-spatially located with the outer edge of the nose-inner arc of Fig. 2 and it would imply a shock with Mach number $M=1.7_{-0.2}^{+0.4}$ as from the Rankine-Hugoniot density jump conditions. To confirm the shock nature of the edge, we extracted and fitted spectra downstream and upstream the front, obtaining temperatures of $k T_{d}=8.2_{-1.3}^{+2.3} \mathrm{keV}$ and $k T_{u}=3.2_{-1.2}^{+2.3}$ $\mathrm{keV}$, respectively. In this case, the Rankine-Hugoniot temperature jump conditions provide a Mach number of $M=2.4_{-0.8}^{+1.1}$, consistent with that derived from the surface brightness analysis. The Mach number that we measured is in agreement with the value of $2.1 \pm 0.1$ reported in Mazzotta et al. (2011). 
In Section 4.2.2, we measured a spectral index $\alpha=1.5 \pm 0.1$ in the region of the nose close to the X-ray detected shock. For the consideration of the previous section this value can be considered as the integrated spectral index of particles that are accelerated via DSA by a shock moving outwards, and then reach the equilibrium with energy losses as in the continuous injection model (Kardashev 1962). The corresponding Mach number is given by equation (9) that implies $M=2.2 \pm 0.2$. This value is consistent with the one derived from the X-rays.

The detection of a shock front coincident with a radio relic strongly supports the relic-shock connection and allows to evaluate the efficiency of particle acceleration in cluster outskirts. Recently, Botteon et al. (2019) studied the acceleration efficiency in a sample of relics, including RXC J1314.4-2515, and concluded that DSA from the thermal pool is severely challenged by the large acceleration efficiency required to reproduce the observed relic luminosity.

The sector chosen for X-ray analysis maximizes the surface brightness jump that bounds the radio emission of the inner arc of the relic and is part of the ' $M$ ' shape structure observed by Mazzotta et al. (2011). Conversely, the outer arc of the relic lies in front of this feature, which is also located in a region where the X-ray brightness is too low for a proper characterization in surface brightness. The presence of this feature and of a possible, still undetected, underlying shock supports the idea of a complex merger dynamic.

\subsection{Particle re-acceleration in the radio halo}

The radio halo in RXC J1314.4-2515 is not a classical giant (i.e. $\geq 1 \mathrm{Mpc}$ ) radio halo since its extension reaches a maximum of $550 \mathrm{kpc}$ in the W-E direction. Cassano et al. (2007) found a scaling relation between the size of radio haloes and their radio power. This relation is motivated in the framework of the re-acceleration scenario in which relativistic electrons are re-energized in situ by mechanisms associated with the turbulence originated during cluster merger (Brunetti et al. 2001; Petrosian 2001). Using their relations, we found that, for the halo radio power at $1.4 \mathrm{GHz}$, listed in Table 3 , the expected halo radius, defined as $\sqrt{R_{\min } \times R_{\max }}$, $R_{\min }$ and $R_{\max }$ being the minimum and maximum radii measured on the $3 \sigma$ radio contours, is $409_{-35}^{+26} \mathrm{kpc}$. The average radius of the halo in RXC J1314.4-2515 is instead $250 \mathrm{kpc}$. Hence, this halo is slightly smaller than what would be expected. This is not an observational effect due to missing short baselines since with the JVLA D configuration it is possible to recover emission on a scale of several Mpc at the cluster redshift, if present.

The radio emission permeating the central region of RXCJ1314.4-2515 is spatially connected to the $\mathrm{W}$ radio relic although they show different spectral index trend and polarization properties. The halo emission shows a smooth spectral index distribution with an average value of $1.3 \pm 0.2$ (Fig. 6). Instead, the radio relic shows a typical steepening of the spectrum in the downstream region. The spectral index profile of the whole western extended emission (see Fig. 8) is similar to the one observed in the Toothbrush radio relic by van Weeren et al. (2016) and Rajpurohit et al. (2018), with a steepening between the relic and the halo, followed by flattening of the spectrum. This spectral index distribution suggests a link between different mechanisms of particle acceleration. The flattening of the spectral index in the halo region may be due to the re-acceleration of aged electrons previously accelerated by the shock in the $\mathrm{W}$ relic. If this is the case, this cluster indicates that the seed electrons necessary for turbulent re-acceleration to work could be supplied by shock waves that also inject turbulence in the cluster core (van Weeren et al. 2016).

It is well established that the radio power of giant radio haloes scales with the cluster X-ray luminosity, and thus with the cluster mass (e.g. Feretti et al. 2012). The Sunyaev-Zel'dovich effect has also been used as a proxy of the cluster mass to derive a well-defined scaling relation between the radio power and cluster mass (Cassano et al. 2013). The radio halo hosted in RXC J1314.4-2515 follows this relation. If we consider the halo emission as detached from the relic on the basis of the spectral index profile, the radio halo luminosity at $1.4 \mathrm{GHz}$ is $1.16 \pm 0.07 \cdot 10^{24} \mathrm{~W} \mathrm{~Hz}^{-1}$ (see Table 3) while the one expected from the correlation is $2.2_{-0.5}^{+0.7} \times 10^{24} \mathrm{~W} \mathrm{~Hz}^{-1}$. Despite its small size, the radio power originating from this radio halo is in agreement within the $3 \sigma$ confidence level of the correlation.

\subsection{Projection effects and polarization}

Although projection effects are expected to be minimal in double relic clusters, they are probably playing a role in the observed properties of this system. Golovich et al. (2018) derived that RXC J1314.4-2515 has a large line-of-sight velocity difference between the merging clusters, which they found is atypical for double relic systems. The viewing angle of the merger $\theta$ (i.e. the angle between the line of sight and the merger axis) was constrained by means of a comparison with simulated cluster by Wittman et al. (2018). They found that $\theta>37^{\circ}$ within the 68 per cent confidence interval.

In the absence of optical observations, polarization data can also be used to get an independent constraint on the merger axis. Ensslin et al. (1998) developed a simplified relation that links the observed average polarization of a radio relic with its viewing angle, given its integrated spectral index. This relation is derived under a number of assumptions and it does not take into account the effects due to beam and Faraday depolarization. In spite of this, it could be used to derive a lower limit on the viewing angle (e.g. Hoang et al. 2018).

Referring to the $S$-band measurements, in the northern part of the E relic the average polarization fraction is $41 \pm 6$ per cent and $\alpha=1.2 \pm 0.2$. This implies a viewing angle of $\sim 62^{\circ}$. For the $\mathrm{W}$ relic we considered only the northern arc, since the southern nose of the shock is experiencing stronger internal Faraday depolarization as discussed in Section 6.6. The arc is polarized on average at $31 \pm 3$ per cent and we can use a spectral index of $\alpha=1.5 \pm 0.1$ as derived at the edge of the relic: the viewing angle is therefore $\sim 55^{\circ}$. A value of around $60^{\circ}$ can thus be considered as a lower limit for the viewing angle of the merger.

With the fractional polarization value of relics we can also derive the Mach number of the underlying shock as done in Kierdorf et al. (2017). Here, the polarization is derived for relics seen perfectly edge-on assuming a weak upstream magnetic field that is tangled and compressed on small scales at the shock front. Under these assumptions, a fractional polarization of $41 \pm 6$ per cent corresponds to a Mach number of $\sim 1.7$ for the $\mathrm{E}$ relic while for a fractional polarization of $31 \pm 3$ per cent, as in the $W$ relic arc, $M \sim 1.4$. These values are both lower than the Mach numbers that we estimated from the spectral index and X-ray analysis. This supports the idea that projection effects may lower the observed fractional polarization of the relics.

\subsection{Comparison with a simulated radio relic}

In the following, we compare our results with mock X-ray and radio images of a simulated radio relic. The $3 \mathrm{D}$ cubes of simulated 
quantities can be integrated along different directions, changing the angle $\theta$ and obtaining projected images of the simulated cluster at different viewing angles. This comparison allows us to (i) check if the values of RM obtained in the simulation can be compared to the observed ones, with the aim of deriving a rough estimate of the magnetic field in the region of the diffuse radio emission and to constrain the electron acceleration efficiency at the shock (ii) test the viewing angle using realistic geometrical conditions and introducing the effects of beam depolarization and Faraday rotation, both external and internal.

We used a high-resolution cosmological magnetohydrodynamical simulation of a galaxy cluster produced with the adaptive mesh refinement code ENZO (Bryan et al. 2014), as in Vazza et al. (2018). The cluster has a virial mass of $M_{100} \approx 1.3 \times 10^{15} \mathrm{M}_{\odot}$ at $z=0.05$. The maximum spatial resolution in the simulation is $3.95 \mathrm{kpc}$ per cell. However, in the region where a simulated radio relic is used for a comparison with our observations the effective resolution is $7.9 \mathrm{kpc}$ per cell.

At the beginning of the simulation $(z=30)$, we assumed a uniform magnetic field value $B_{0}=0.1 \mathrm{nG}$ along each coordinate axis, which mimics a primordial magnetic field and is below the upper limits from the analysis of the cosmic microwave background (e.g. Subramanian 2016). The field is then amplified in the course of the cluster formation process, both due to gas compression (for $z>1$ ) and small-scale dynamo (for $z \leq 1$ ) and finally reaches a typical magnetic field strength of $\sim 2-5 \mu \mathrm{G}$ in the cluster core, and of $\sim 0.1-0.3 \mu \mathrm{G}$ at the cluster virial radius, showing a tangled $3 \mathrm{D}$ magnetic field with a typical scale of $\sim 50 \mathrm{kpc}$ (see DomínguezFernández et al. 2019 for more details on the simulation).

We followed an approach similar to Wittor et al. (submitted) to compute the polarized emission of the simulated radio relic. Therefore, we used a velocity jump method as in Vazza, Brunetti \& Gheller (2009) to detect and flag shock waves in the simulation and for each flagged cell, we computed the integrated polarized emission following the model by Burn (1966, equation 1). We follow the formalism of Hoeft \& Brüggen (2007, equation 29) to compute the downstream radio profile of an electron population injected at the shock via DSA and that cools via synchrotron and Inverse Compton. We evaluated the profile at nodes on the simulation grid along the shock normal. For the frequency range probed in this work, the electron cooling length is of a few kpc and hence most profiles are computed within one simulation cell. Therefore, we assume a homogeneous magnetic field and downstream temperature for each profile. Analogous, we compute the downstream profiles of the perpendicular and parallel component of the radio emission, that determine the intrinsic degree of polarization (see equation 63.7 in Rybicki \& Lightman 1986).

In order to match the observed radio power of RXCJ1314.4-2515 the acceleration efficiency by Hoeft \& Brüggen (2007) is not enough for this weak shock. Following Vazza et al. (2015), we included also the effect of re-accelerated particles by shocks, which is expected to dominate over the contribution from freshly injected particles for $\mathcal{M} \leq 3$ shocks (e.g. Pinzke et al. 2013). We include both internal and external Faraday rotation, directly using the three-dimensional magnetic field from the simulation.

We selected a galaxy cluster (SIM for short) in a post-merger state at redshift 0.05 . We found that the $2 \mathrm{D}$ projection of this cluster at a viewing angle of $70^{\circ}$ matches the observed properties: (i) the selected cluster shows a shock front in the X-rays, with an ' $M$ ' shaped morphology, similar to the one detected in RXC J1314.4-2515, (ii) a radio relic $\sim 900 \mathrm{kpc}$ long traces the
Table 5. Quantities of interest in the comparison between the observed RXC J1314.4-2515 (RX1314) and the simulated cluster (SIM). Column 1: cluster name; Column 2: radio power at $3 \mathrm{GHz}$. The flux density was extracted from a region of the same physical size (i.e. $\sim 1.5 \times 10^{5} \mathrm{kpc}^{2}$ ) in both clusters; Column 3: the X-ray luminosity of RXC J1314.4-2515 from Piffaretti et al. (2011) (given without uncertainties) was used to compute the luminosity of RXC J1314.4-2515 in the range $0.5-2 \mathrm{keV}$ using WebPIMMS (https://heasarc.gsfc.nasa.gov/cgi-bin/Tools/w3pimms/ w3 pimms.pl). The luminosity of SIM was computed as the sum in the whole cube; Column 4: thermal electron density at the shock front. From the density profile derived in Mazzotta et al. (2011) we listed the post-shock electron density with an uncertainty derived from their profile. For SIM we computed an average value in the post-shock region and we used the standard deviation as uncertainty. Column 5: Mach number of the shock. We list the value derived in Section 6.2 for RXC J1314.4-2515. For SIM, we computed an average value in the cells within the shock region where $M$ $>2$, and we used the standard deviation of the distribution as uncertainty.

\begin{tabular}{lcccc}
\hline Cluster & $\begin{array}{c}\left.P_{3} \mathrm{GHz}^{-1}\right) \\
\left(10^{23} \mathrm{~W} \mathrm{~Hz}^{-1}\right)\end{array}$ & $\begin{array}{c}L_{\mathrm{X}} \\
\left(10^{44} \mathrm{erg} \mathrm{s}^{-1}\right)\end{array}$ & $\begin{array}{c}n_{\mathrm{e}} \\
\left(10^{-3} \mathrm{~cm}^{-3}\right)\end{array}$ & $M$ \\
\hline RX1314 & $11.5 \pm 0.6$ & 8.91 & $0.7 \pm 0.05$ & $1.7_{-0.2}^{+0.4}$ \\
SIM & $11.1 \pm 0.4$ & 5.65 & $3.7 \pm 0.9$ & $2.2 \pm 0.2$ \\
\hline
\end{tabular}

shock front at a distance of $\sim 500 \mathrm{kpc}$ from the X-ray centroid, with a region of the relic lying beyond the bulk of the shock, where the $\mathrm{X}$ ray luminosity decreases, (iii) the average Mach number at the shock is similar to the one detected in RXC J1314.4-2515. The main characteristics of the simulated cluster are listed in Table 5, with the same quantities estimated for RXC J1314.4-2515 for comparison. The 2D projected gas density and maximum magnetic field strength along each line of sight are shown in Fig. 15. The simulated magnetic field shows filamentary and complex sub-structures. The average width of magnetic field filaments in Faraday space is few tens rad $\mathrm{m}^{-2}$ thus they cannot be resolved at the maximum resolution in Faraday space that we reach combining $L$ - and $S$-band data.

By comparing the simulated radio power from the relic at $3 \mathrm{GHz}$ to the observed one of RXC J1314.4-2515, we find that an electron acceleration efficiency of $\xi_{\mathrm{e}} \geq 1000$ per cent (referred to the kinetic energy flux of shocked cells) is needed, meaning that the kinetic energy of this shock would not be enough to explain our $3 \mathrm{GHz}$ observation of RXC J1314.4-2515. This value obviously requires something beyond DSA, and as argued in other works it can be attained only if a pre-existing population of fossil electrons (with $\gamma$ $=10^{2}-10^{3}$ ) was present upstream of the merger shock that produced the western radio relic in RXC J1314.4-2515 (Markevitch et al. 2005; Pinzke et al. 2013; Kang \& Ryu 2015; Botteon et al. 2016a; Eckert et al. 2016a). Our modelling suggests that this is reasonable, provided that the injection of fossil electrons happened no longer than $\sim 1$ Gyr before the $M \approx 2$ shock crosses the region where the western radio relic is now visible.

The origin of such fossil population is not clear as it could be for the E relic. It could originate from an AGN that is no longer active or from previous shocks or turbulence generated in the cluster formation process. Considering that the typical timescale for turbulent acceleration larger than 1 Gyr on scales of $500 \mathrm{kpc}$ (Brunetti \& Jones 2014) the observed radio halo cannot have supplied already aged electron in the region where the $\mathrm{W}$ relic is observed. Fossil electrons have $\gamma=10^{2}-10^{3}$ and to detect them and discover their origin we would need low-frequency observation in the $\mathrm{MHz}$ regime.

Since the average synchrotron power of a relativistic electron population is $\langle P\rangle \propto \gamma^{2} B^{2}$ (if $B \leq 3.2 \mu \mathrm{G}(1+z)^{2}$ as in our case here), 

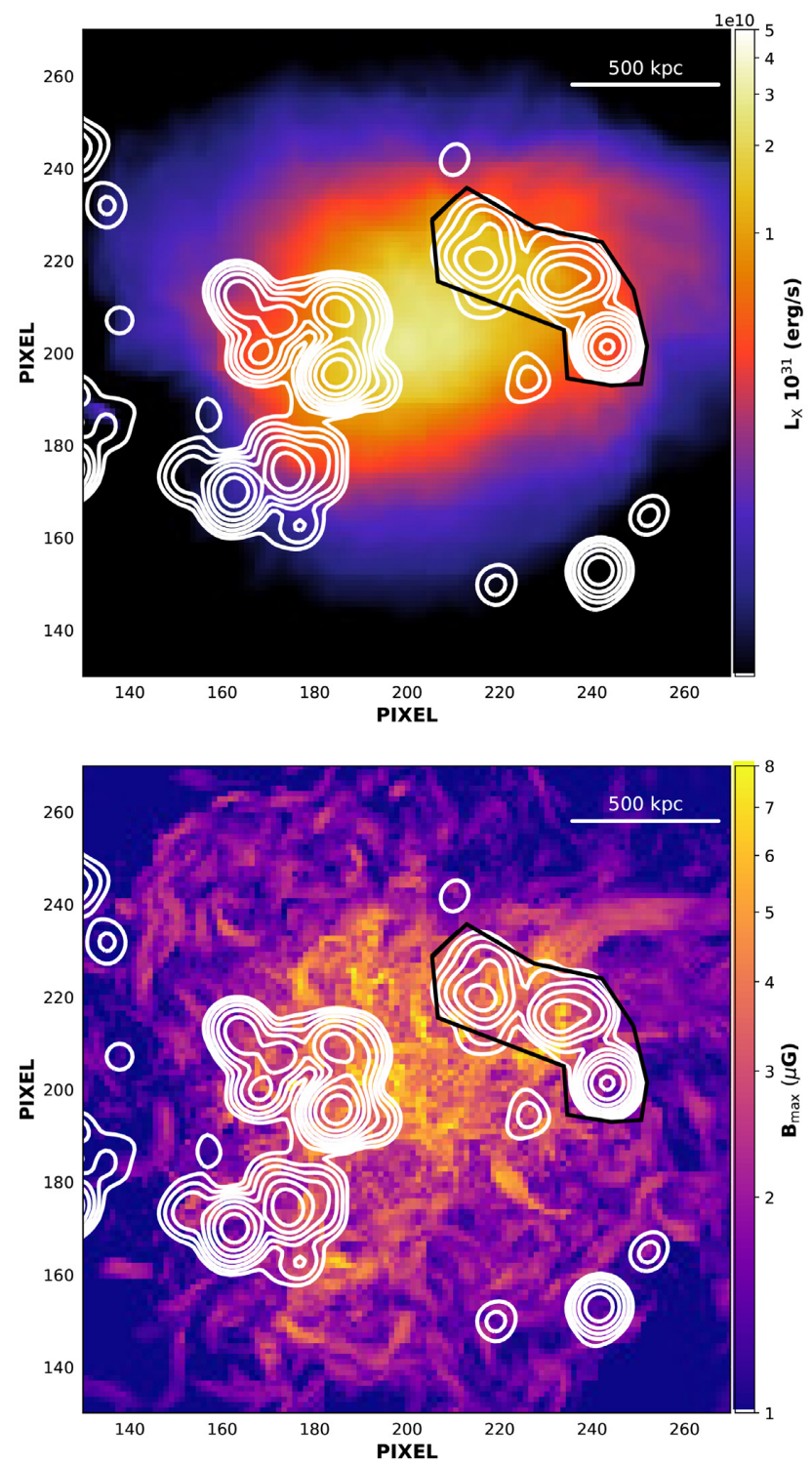

Figure 15. Top panel: X-ray luminosity map of the simulated cluster (SIM), with $3 \mathrm{GHz}$ radio contours overlaid. Contours start at $1.8 \times 10^{20} \mathrm{~W} \mathrm{~Hz}^{-1}$ and are spaced by a factor of 2 . The viewing angle is $70^{\circ}$. The resolution of the image is the same of the simulation (i.e. $15.8 \mathrm{kpc} \mathrm{pixel}^{-1}$ ) while the radio power image was smoothed with a Gaussian kernel of $\sim 80 \mathrm{kpc}$. Bottom panel: maximum magnetic field along each line of sight (pixel) of the 3D simulated cluster for a $70^{\circ}$ viewing angle. Radio contours are the same as in the top panel. In both panels, the black region is the one used to extract the relic radio power.

in principle either a higher magnetic field, or a larger acceleration efficiency (and/or a higher density of seed fossil electrons) are needed to match the observation. However, as we shall see, the polarization properties of the west relic can be used to constrain the magnetic field amplitude, which in turn sets a lower limit on the amount of necessary particle re-acceleration (or higher efficiency).

We performed RM synthesis on the simulated data. We obtained Stokes $I, Q$ and $U$ 2D projections in the same frequency range and sub-bands of our measurements in the $S$ band. We smoothed all images with a Gaussian kernel of the physical size of the restoring beam of the $S$-band image (i.e. FWHM $\sim 80 \mathrm{kpc}$ ). With the same procedure described in Section 5.3 we obtained images
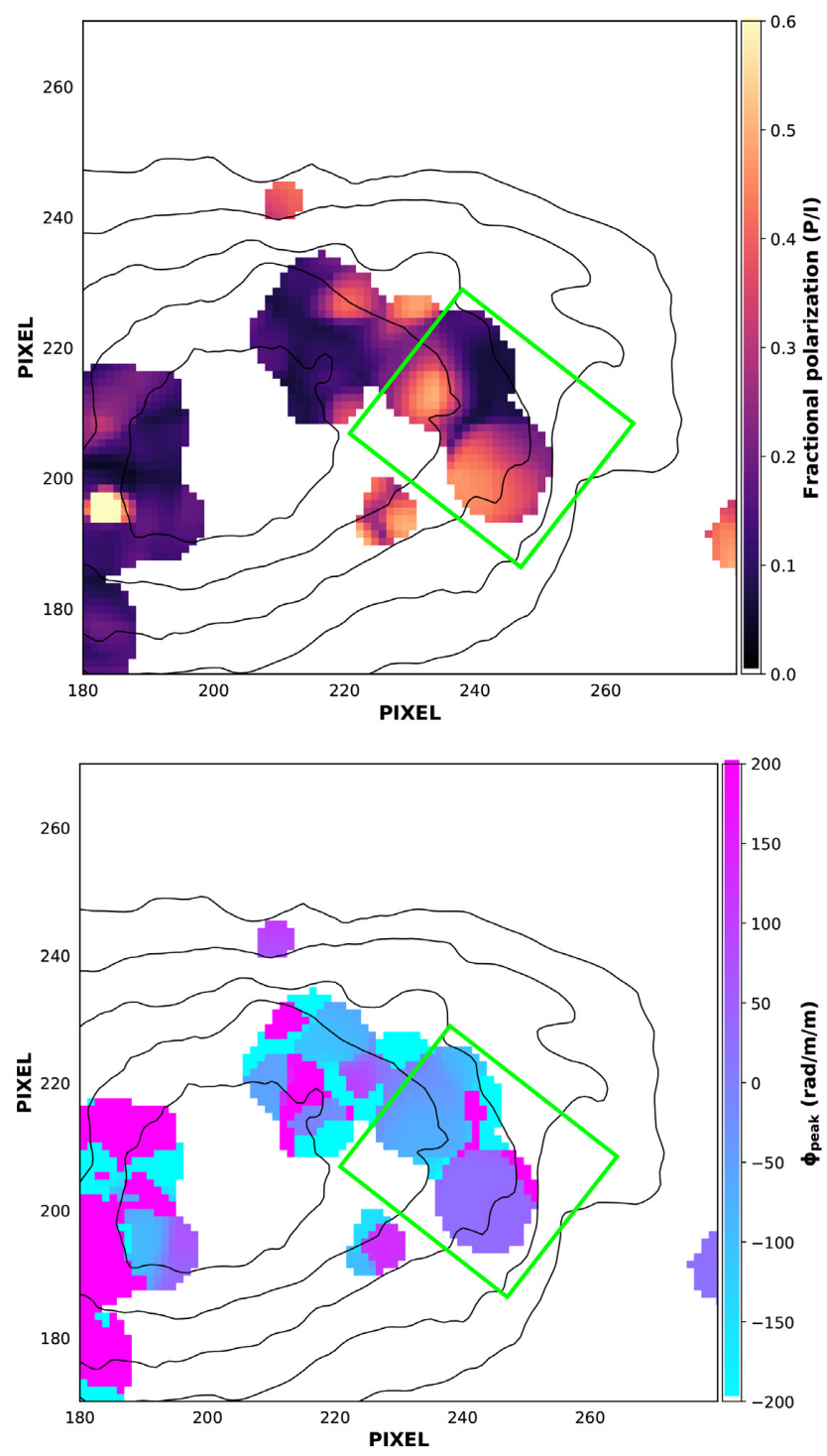

Figure 16. Fractional polarization (top panel) and Faraday depth (bottom panel) of the radio diffuse emission in SIM in the $2-4 \mathrm{GHz}$ band. A threshold is imposed to have the same total intensity dynamical range of our observation in the region of the relic. Black contours are from the X-ray luminosity image in the energy band $0.5-2 \mathrm{keV}$ : they start at $10^{40} \mathrm{erg} \mathrm{s}^{-1}$ and they are spaced by a factor of 2 . The green box shows the region where we extracted the average fractional polarization and $\left|\phi_{\text {peak }}\right|$ values.

of $\phi_{\text {peak }}$ and $P=\left|\widetilde{F}\left(\phi_{\text {peak }}\right)\right|$ and we obtained the linear degree of polarization map by dividing the polarization and total intensity smoothed images. Fractional polarization and $\phi_{\text {peak }}$ images for the 2D projection at $\theta=70^{\circ}$ are shown in Fig. 16. In all the images we imposed a threshold in order to obtain the same dynamical range of our observations in total intensity.

Also from the simulation we obtained Faraday-complex spectra in some regions of the relic. In order to produce a high-resolution Faraday spectrum of the simulated relic, we also performed the RMsynthesis on the simulated data between 1 and $4 \mathrm{GHz}$ reaching a resolution of $37 \mathrm{rad} \mathrm{m}^{-2}$ as in the $S+L$-band measurement. The highresolution spectrum of a representative pixel is shown in Fig. 17. The Faraday spectrum is shown together with the clean components found during the RM clean procedure and the final cleaned Faraday spectrum. Several filaments are clearly separated after the RM- 


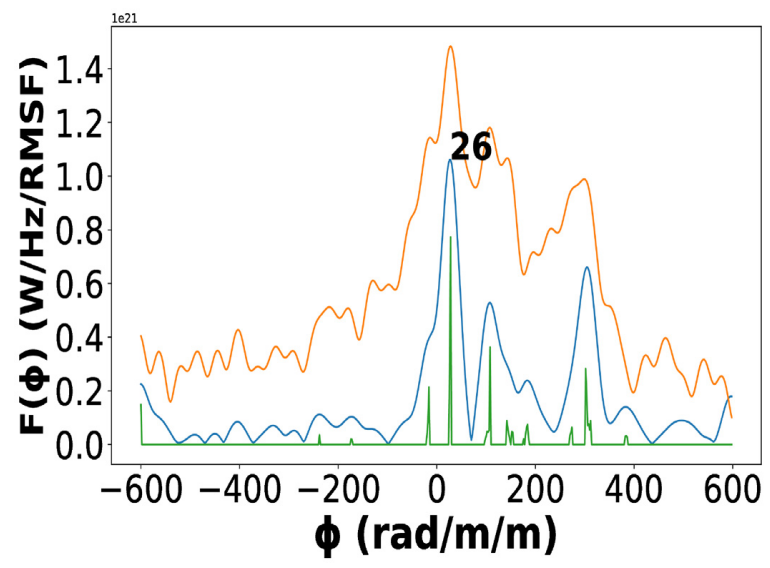

Figure 17. Same image as the bottom-right panel of Fig. 13 but for the simulated radio relic.

clean. The filaments in the simulation appear to be spread over $\sim 300 \mathrm{rad} \mathrm{m}^{-2}$ in Faraday depth and they have different luminosity.

We can use as a benchmark the average fractional polarization and the distribution of $\phi_{\text {peak }}$ values in the most external region of the relic, marked with the green box in Fig. 16. We chose a region where we obtained Faraday-simple spectra in the $S$ band as in the northern arc of the W relic of RXC J1314.4-2515, thus we can more easily compare the results of the RM synthesis. The average fractional polarization for $\theta \sim 70^{\circ}$ in the smoothed image is 32 per cent and it is consistent with what we obtained from the observation at the same physical resolution (i.e. $31 \pm 3$ per cent in the western relic). This value takes into account the depolarization due to the smoothing of the polarization vectors on physical scales between 15 and $80 \mathrm{kpc}$ since the simulated magnetic field has important sub-structure on such scales.

In SIM, we found higher values of $\phi_{\text {peak }}$ compared to the $\phi_{\mathrm{cl}}$ values obtained in RXC J1314.4-2515 in the $S$ band, but the thermal electron density in the region of the shock is on average $\sim 5$ times lower in RXC J1314.4-2515 than in SIM (see Table 5). To make a fair comparison on the effect of the magnetic field on the Faraday rotation in the two clusters, we compare in Fig. 18 the distribution of Faraday depths obtained in the simulation reduced by a factor of 5 with the observed one (foreground subtracted). The average and median values of the two distributions are consistent within the uncertainties. This indicates that the magnetic field in the relic region in $\mathrm{RXCJ1314.4-2515}$ is already of the order of the simulated one (i.e. $\sim 1 \mu \mathrm{G}$ ), and hence that only a higher (re)acceleration efficiency can explain the power radio emission from the $\mathrm{W}$ relic. We notice that while the role of re-accelerated fossil electrons has been already proposed as key ingredient to explain observed radio relics (e.g. Pinzke et al. 2013; Kang \& Ryu 2015, 2016), this is the first time in which we can constrain its importance by simultaneously fixing the uncertainties on the magnetic field in the relic region, thanks to polarization data and simulations.

In spite of similar median values, the two distributions in Fig. 18 are very different. For this comparison we used the peak values of $S$-band spectra in the green region of Fig. 16, where Faraday spectra are not resolved and show a single component. The simulated distribution is thus dominated by the emission of the brightest filament at a certain Faraday depth and the narrower distribution compared to the data is due to the fact that the same filament dominates in the sampled spatial region. The broader distribution

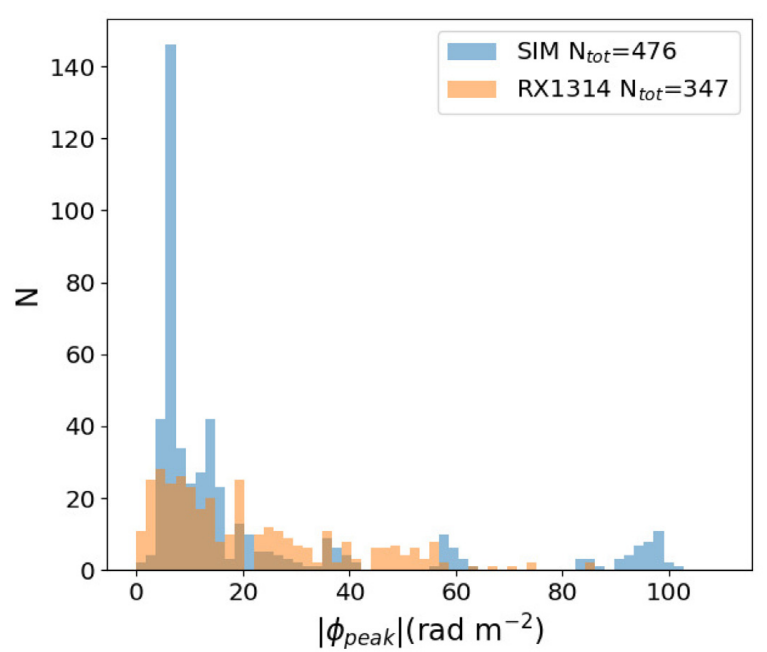

Figure 18. Distribution of Faraday depths. The $\left|\phi_{\text {peak }}\right|$ values of the simulation were reduced by a factor 5 to be compared with the observation, due to the different thermal electron density. For the observation (RX1314), the values are obtained in the northern region of the $\mathrm{W}$ relic arc in the $S$-band measurement. For the simulation (SIM) the values are extracted in the region marked with the green box in Fig. 16.

of RM values found in the northern arc of the radio relic shows that various emitting structures at different Faraday depths are cospatially located in a region equivalent to the one considered in the simulation.

\subsection{Faraday-complex emission}

As reported in Section 5.3.3, we observed Faraday-complex emission in the nose of the $\mathrm{W}$ relic. This means that linearly polarized emission originates from different layers of the radio relic that experienced different amounts of Faraday rotation. Faraday-complex structures were observed also in the Toothbrush radio relic (van Weeren et al. 2012). In this region, we observed features in the Faraday dispersion functions spread over a range of 150-250 rad $\mathrm{m}^{-2}$.

As an example, in Fig. 13, the $S$ - and $S+L$-band Faraday dispersion function (i.e. Faraday spectrum) is shown for two pixels. The first row shows a Faraday-simple source (i.e. a pixel at the position of the source labelled with $\mathrm{C}$ in Fig. 1), the second row a Faraday-complex one (i.e. a pixel in the nose region).

The RM clean found a single component in the spectrum of the Faraday-simple source, both with the $S$ - and $S+L$-band resolution. In the $S$ band, the Faraday-complex pixel is observed as a single peak but with an FWHM slightly larger than the RMSF resolution (i.e. $\delta \phi=188 \mathrm{rad} \mathrm{m}^{-2}$ ): some cleaned components are found, within a range of $200 \mathrm{rad} \mathrm{m}^{-2}$. Instead, in the $S+L$-band measurement, where we have higher resolution in Faraday space, the RM clean on the Faraday-complex spectrum found several components, with a similar flux density, and a width of $150 \mathrm{rad} \mathrm{m}^{-2}$.

The shape of a complex Faraday spectrum is indicative of the medium generating the emission. A single broad feature in the Faraday-spectrum originates from a regular distribution of magnetic fields and thermal electron density while a series of peaks are expected if filamentary magnetic field structures are overlaid along the same line of sight. In both cases, the Faraday rotation occurs in the emitting region. Our simulation suggested that we should expect a series of peaks in our case since the magnetic field has filamentary 


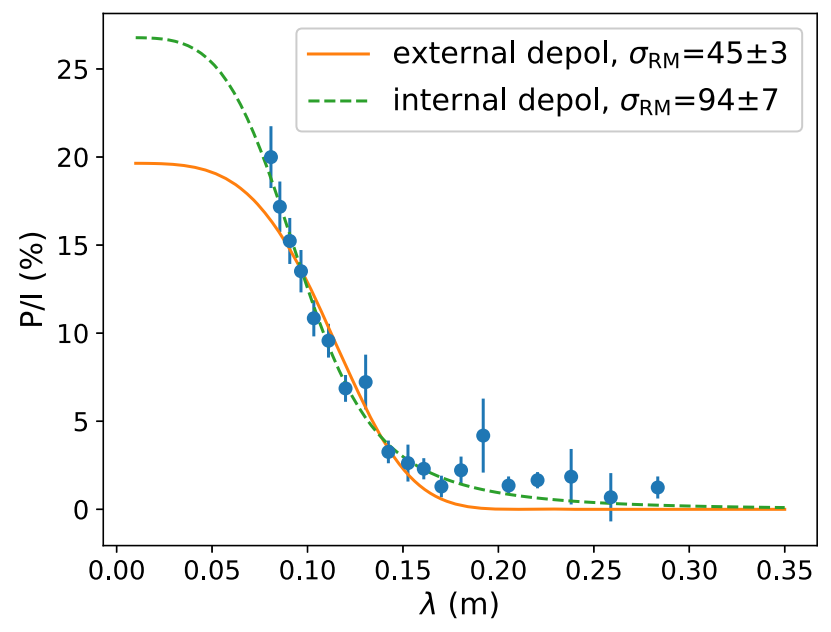

Figure 19. Depolarization profile of the nose of the western relic. Fractional polarization was computed in each sub-band separately. Green dashed line is the fitted internal depolarization model while the orange line is the external depolarization model.

structures with a width in Faraday space lower than $40 \mathrm{rad} \mathrm{m}^{-2}$ (see Figs 15 and 17).

Although the RM clean procedure helps in reducing secondary lobes when a peak is detected at high signal-to-noise ratio, it is not well suited to detect Faraday-complex sources, since it assumes a thin model for every detected component, and it easily diverges at lower signal-to-noise values. Furthermore, the performance of RM synthesis in detecting Faraday-complex sources strongly depends on the frequency coverage of the observation. Some techniques have been proposed to overcome such problems and to optimize the reconstruction of Faraday-complex spectra (Frick et al. 2010; Li et al. 2011). All of them assume a model for the synchrotron-emitting and Faraday rotating medium, and nowadays their performances are still tested and debated (see e.g. Sun et al. 2015; Miyashita, Ideguchi \& Takahashi 2016; Schnitzeler 2018; Ideguchi, Miyashita \& Heald 2018 , for the comparison of RM clean with other methods).

On the basis of the spectrum shown in the bottom-right panel of Fig. 13 it is thus hard to distinguish between a single broad feature or a convolution of several Faraday-simple spectra originating from different filaments. For this reason we also studied the wavelength dependence of the degree of polarization. If filamentary and complex structures of magnetic field originate the complex Faraday spectrum, internal Faraday depolarization is expected (Arshakian $\&$ Beck 2011). The dispersion of RM values that we detected in our unresolved structures should be the same as the turbulent dispersion causing the depolarization, $\sigma_{\mathrm{RM}}$.

We imaged the $Q$ and $U$ data cubes between 1 and $4 \mathrm{GHz}$ into 20 frequency sub-bands of $150 \mathrm{MHz}$ each and we smoothed the data to 30 arcsec resolution to increase the signal-to-noise ratio. We calculated the fractional polarization of each sub-bands separately. The depolarization trend computed in the region of the nose, marked with a green circle in the top panel of Fig. 11, is shown in Fig. 19. We fitted both internal and external Faraday depolarization as modelled in Arshakian \& Beck (2011). Both depend on RM dispersion, $\sigma_{\mathrm{RM}}$, and wavelength. Internal Faraday depolarization well represents our data with a $\sigma_{\mathrm{RM}}$ of $94 \pm 7 \mathrm{rad} \mathrm{m}^{-2}$. This shows that the depolarization is occurring in the same emitting volume. We note that the dispersion value is of the same order of magnitude of the range of Faraday depths that we detected with the RM synthesis.
Several magnetic field filaments along the line of sight in a turbulent ionized gas are likely the origin of the Faraday-complex structures and of depolarization in the nose region.

We studied the depolarization trend also in the northern region of the E relic, marked with the green circle in the upper panel of Fig. 11. In this case, the RM dispersion for internal and external Faraday depolarization are $12 \pm 2$ and $7 \pm 1 \mathrm{rad} \mathrm{m}^{-2}$, respectively, and the reduced $\chi^{2}$ is 1.2 for both. Under the simplified assumption that the magnetic field is the same at the position of the two relics, the ratio of $\sigma_{\mathrm{RM}}$ is equal to the ratio of the thermal electron densities along the line of sight (see e.g. the definition of $\sigma_{\mathrm{RM}}$ given in Arshakian $\&$ Beck 2011). This would imply that the thermal electron density in the region of the E relic is $0.13 \pm 0.02$ or $0.16 \pm 0.02$ times lower than in the $\mathrm{W}$ relic region, considering only internal or external depolarization, respectively. Overall this is in agreement with the upper limit from the X-rays and with the estimate made in Section 6.1. Anyway, simulations probed that every shock has a peculiar and complex morphology that does not allow a simplified and general description (Hoeft et al. 2011).

When the Faraday spectrum is unresolved, the peak value of the spectrum represents the total polarized flux density of the source in $\mathrm{Jy} \mathrm{beam}^{-1}$, integrated along the Faraday spectrum. Conversely, in the case of a resolved Faraday-complex emission, the peak value represents only the main component of the spectrum, and it is a fraction of the total emission of the source. As a result, the polarized flux density of Faraday-complex structures estimated from the main peak is lower than the integrated one, and the fractional polarization that we compute with the RM synthesis should be regarded as a lower limit.

For the purpose of this paper, we decided to use the information that we obtained directly from the RM synthesis technique as it is implemented in PYRMSYNTH. In the pixels where we observed a Faraday-complex spectrum, we considered as integrated polarization that one obtained from the peak of the $S$-band data, where the broad feature is only partially resolved. In this way, we have the best possible estimate for the polarized flux density. Instead, we used $S+L$-band data to recover the RM value of the brightest component $\left(\phi_{\text {peak }}\right)$ with higher precision. This value should be used with caution for Faraday-complex spectra.

Even if we were able to recover the true Faraday-complex spectrum of a source, its physical interpretation would not be unique. In fact, there are several distributions of magnetic field, thermal electron density and physical depth that can generate the same observed spectrum. Also beam depolarization should be considered since it could introduce spatially varying $\phi$ components that may contribute to the complexity of the Faraday spectrum. We plan to further study this aspect applying the RM synthesis technique in simulation of cluster radio diffuse emission, taking into account both external and internal Faraday depolarization. The necessary frequency coverage and appropriate RM synthesis methods for reconstructing such complicated Faraday spectrum could be also investigated in the comparison with synthetic Faraday-complex spectra.

\section{CONCLUSIONS}

In this paper, we performed a detailed study of the radio emission of the merging galaxy cluster RXC J1314.4-2515 in the frequency range 1-4 GHz. We exploited a variety of JVLA observations: full polarization A, B, C, and D configurations between 1 and $2 \mathrm{GHz}$ ( $L$ band) and DnC configuration archival data in the $2-4 \mathrm{GHz} S$ band. We performed both spectral analysis and RM synthesis on 
the entire cluster field, and in particular on two radio relics and the central radio halo. This study allows us to investigate possible scenarios for the origin of the extended radio emission observed in RXC J1314.4-2515.

Our results can be summarized as follow:

(1) New multiconfigurations JVLA data offer unprecedented view of RXC J1314.4-2515 at 1.5 GHz. We detected the two relics and the central radio halo. The western relic shows interesting substructures: two arcs depart from the X-ray detected shock, one of the two being $\sim 900 \mathrm{kpc}$ long and extending towards the outer region of the cluster. The eastern relic embeds a NAT radio galaxy, member of the cluster.

(2) This study revealed a possible connection between the AGN activity and the extended emission of the E radio relic. The spectral index profile supports a re-acceleration scenario in which a seed population of mildly relativistic particles, pre-accelerated by AGN jets, are subsequently re-accelerated by merger driven shock waves. Polarization properties of the entire eastern system suggest that the shock waves were able to align the magnetic field lines along the shock plane, but we did not find Faraday rotation caused by the cluster magnetic field in this region. Only very deep X-ray observations could proof the presence of a shock underlying the eastern radio relic.

(3) Spectral index profiles and polarization properties, allowed us to disentangle the contributions of the relic and the halo to the western diffuse emission. The relic shows a typical spectral steepening downstream of the X-ray detected shock, while a different acceleration process should be able to re-energize particles in the radio halo region. The polarization properties support this scenario: only the radio relic is detected in polarized intensity at $3 \mathrm{GHz}$.

(4) We discovered Faraday-complex emission in the northern region of the $\mathrm{W}$ relic. This indicates the presence of thermal gas and complex magnetic field morphology within the relic volume. The fractional polarization in the region is low (i.e. on average $8.5 \pm 0.6$ per cent at $3 \mathrm{GHz}$ ) due to internal Faraday depolarization. We obtained very different RM dispersion from the depolarization trend of the two relics. However, deriving constraints on underlying magnetic field would require a more detailed knowledge of the gas thermal density, projection effects, and shock-wave morphology.

(5) We studied the X-ray shock coincident with the inner arc of the western radio relic. We derived a Mach number consistent with that found by Mazzotta et al. (2011) (i.e. $M=1.7_{-0.2}^{+0.4}$ from the Xray surface brightness jump and $M=2.4_{-0.8}^{+1.1}$ from the temperature jump). The Mach number derived from the radio spectral index assuming DSA mechanism is consistent with the one derived from the X-ray analysis.

(6) By comparing our observation with a cosmological simulation, we tested a possible viewing angle of $\sim 70^{\circ}$. The consistency check pointed out that the simulation is able to reproduce the observed fractional polarization and the RM of the western relic with a magnetic field of $\sim 1 \mu \mathrm{G}$ in the relic region and important substructures on scales between 15 and $80 \mathrm{kpc}$. The required electron acceleration efficiency to match the observed radio power provides the need of significant re-acceleration of fossil electrons.

In the future, to resolve and interpret Faraday-complex structures, we could exploit the SKA-MID, ${ }^{7}$ which will reach a resolution in Faraday space of $\sim 5 \mathrm{rad} \mathrm{m}^{-2}$, spanning the frequency range

${ }^{7}$ https://www.skatelescope.org/ between $350 \mathrm{MHz}$ and $14 \mathrm{GHz}$. A deeper understanding of the physical interpretation of such structures should be also reached thanks to the comparison with simulation (Loi et al. 2018). The combination of radio polarimetric studies, high-resolution spectral index imaging, total intensity radio observation at $50-350 \mathrm{MHz}$, and deep X-ray observations of merger shocks promises to shed new light on particle acceleration processes occurring in the ICM, on the role played by the fossil plasma and on the properties of the magnetic field at merger shocks.

\section{ACKNOWLEDGEMENTS}

$\mathrm{CS}$ and $\mathrm{AB}$ on acknowledge support from the European Research Council starting grant (ERC-StG) DRANOEL, no. 714245. ABon acknowledges support from the Ministero dell'Istruzione dell'Università e della Ricerca (MIUR) grant FARE SMS. CS and ABon acknowledge T. Venturi for useful discussions and for sharing GMRT images. DW and FV acknowledge financial support from the European Union's Horizon 2020 program under the ERCStG MAGCOW, no. 714196. The cosmological simulations were performed with the ENZO code (http://enzo-project.org), which is the product of a collaborative effort of scientists at many universities and national laboratories. The authors gratefully acknowledge the Gauss Centre for Supercomputing e.V. (www.gauss-centre.eu) for supporting this project by providing computing time through the John von Neumann Institute for Computing (NIC) on the GCS Supercomputer JUWELS at Jülich Supercomputing Centre (JSC), under project nos. 11823, 10755, and 9016 as well as hhh42 (P.I. FV) and hhh44 (P.I. DW). ABot thanks D. Eckert for helpful suggestions regarding the XMM-Newton data analysis. Part of this work was performed under the auspices of the U.S. Department of Energy by Lawrence Livermore National Laboratory under Contract DE-AC52-07NA27344. RJvW acknowledges support of the VIDI research program with project no. 639.042.729, which is financed by the Netherlands Organisation for Scientific Research (NWO). We thank the anonymous referee for the useful comments.

\section{REFERENCES}

Ackermann M. et al., 2014, ApJ, 787, 18

Akamatsu H., Kawahara H., 2013, PASJ, 65, 16

Arshakian T. G., Beck R., 2011, MNRAS, 418, 2336

Bell A. R., 1978, MNRAS, 182, 443

Bennett C. L., Larson D., Weiland J. L., Hinshaw G., 2014, ApJ, 794, 135

Böhringer H., Chon G., Kronberg P. P., 2016, A\&A, 596, A22

Bonafede A., Feretti L., Murgia M., Govoni F., Giovannini G., Dallacasa D., Dolag K., Taylor G. B., 2010, A\&A, 513, A30

Bonafede A., Vazza F., Brüggen M., Murgia M., Govoni F., Feretti L., Giovannini G., Ogrean G., 2013, MNRAS, 433, 3208

Bonafede A., Intema H. T., Brüggen M., Girardi M., Nonino M., Kantharia N., van Weeren R. J., Röttgering H. J. A., 2014, ApJ, 785, 1

Botteon A., Gastaldello F., Brunetti G., Dallacasa D., 2016a, MNRAS, 460, L84

Botteon A., Gastaldello F., Brunetti G., Kale R., 2016b, MNRAS, 463, 1534 Botteon A., Brunetti G., Ryu D., Roh S., 2019, preprint (arXiv:1907.00966) Brentjens M. A., de Bruyn A. G., 2005, A\&A, 441, 1217

Briggs D. S., 1995, American Astronomical Society Meeting Abstracts, \#112.02

Brüggen M., Bykov A., Ryu D., Röttgering H., 2012, Space Sci. Rev., 166, 187

Brunetti G., Jones T. W., 2014, Int. J. Mod. Phys. D, 23, 1430007

Brunetti G., Setti G., Feretti L., Giovannini G., 2001, MNRAS, 320, 365

Bryan G. L. et al., 2014, ApJS, 211, 19

Burn B. J., 1966, MNRAS, 133, 67 
Caprioli D., Spitkovsky A., 2014, ApJ, 783, 91

Cassano R., Brunetti G., Setti G., Govoni F., Dolag K., 2007, MNRAS, 378, 1565

Cassano R., Ettori S., Giacintucci S., Brunetti G., Markevitch M., Venturi T., Gitti M., 2010, ApJ, 721, L82

Cassano R. et al., 2013, ApJ, 777, 141

Cornwell T. J., Golap K., Bhatnagar S., 2008, IEEE J. Sel. Top. Signal Process., 2, 647

Dasadia S. et al., 2016, ApJ, 820, L20

de Gasperin F., van Weeren R. J., Brüggen M., Vazza F., Bonafede A., Intema H. T., 2014, MNRAS, 444, 3130

Domínguez-Fernández P., Vazza F., Brüggen M., Brunetti G., 2019, MNRAS, 486, 623

Eckert D., Molendi S., Paltani S., 2011, A\&A, 526, A79

Eckert D., Jauzac M., Vazza F., Owers M. S., Kneib J. P., Tchernin C., Intema H., Knowles K., 2016a, MNRAS, 461, 1302

Eckert D. et al., 2016b, A\&A, 592, A12

Ensslin T. A., Biermann P. L., Klein U., Kohle S., 1998, A\&A, 332, 395

Feretti L., Schuecker P., Böhringer H., Govoni F., Giovannini G., 2005, A\&A, 444, 157

Feretti L., Giovannini G., Govoni F., Murgia M., 2012, A\&AR, 20, 54

Finoguenov A., Sarazin C. L., Nakazawa K., Wik D. R., Clarke T. E., 2010, ApJ, 715, 1143

Frick P., Sokoloff D., Stepanov R., Beck R., 2010, MNRAS, 401, L24

Gabici S., Blasi P., 2003, ApJ, 583, 695

George S. J., Stil J. M., Keller B. W., 2012, Publ. Astron. Soc. Aust., 29, 214

George L. T. et al., 2017, MNRAS, 467, 936

Ghirardini V. et al., 2019, A\&A, 621, A41

Golovich N. et al., 2019, ApJ, 882, 69

Golovich N. et al., 2019, ApJS, 240, 39

Govoni F., Feretti L., 2004, Int. J. Mod. Phys. D, 13, 1549

Guo X., Sironi L., Narayan R., 2014a, ApJ, 794, 153

Guo X., Sironi L., Narayan R., 2014b, ApJ, 797, 47

Ha J.-H., Ryu D., Kang H., 2018, ApJ, 857, 26

Hales C. A., Gaensler B. M., Norris R. P., Middelberg E., 2012, MNRAS, 424,2160

Heald G., 2009, in Strassmeier K. G., Kosovichev A. G., Beckman J. E., eds, IAU Symp. Vol. 259, Cosmic Magnetic Fields: From Planets, to Stars and Galaxies. p. 591

Hoang D. N. et al., 2017, MNRAS, 471, 1107

Hoang D. N. et al., 2018, MNRAS, 478, 2218

Hoeft M., Brüggen M., 2007, MNRAS, 375, 77

Hoeft M., Nuza S. E., Gottlöber S., van Weeren R. J., Röttgering H. J. A., Brüggen M., 2011, J. Astrophys. Astron., 32, 509

Hutschenreuter S., Enßlin T. A., 2019, preprint (arXiv:1903.06735)

Iapichino L., Brüggen M., 2012, MNRAS, 423, 2781

Ideguchi S., Miyashita Y., Heald G., 2018, Galaxies, 6, 140

Jagannathan P., Bhatnagar S., Rau U., Taylor A. R., 2017, AJ, 154, 56

Jones F. C., Ellison D. C., 1991, Space Sci. Rev., 58, 259

Kalberla P. M. W., Burton W. B., Hartmann D., Arnal E. M., Bajaja E., Morras R., Pöppel W. G. L., 2005, A\&A, 440, 775

Kang H., Ryu D., 2011, ApJ, 734, 18

Kang H., Ryu D., 2015, ApJ, 809, 186

Kang H., Ryu D., 2016, ApJ, 823, 13

Kardashev N. S., 1962, Sov. Aston., 6, 317

Kierdorf M., Beck R., Hoeft M., Klein U., van Weeren R. J., Forman W. R., Jones C., 2017, A\&A, 600, A18

Li F., Brown S., Cornwell T. J., de Hoog F., 2011, A\&A, 531, A126

Loi F., Murgia M., Govoni F., Vacca V., Prandoni I., Li H., Feretti L., Giovannini G., 2018, Galaxies, 6, 133

Markevitch M., Vikhlinin A., 2007, Phys. Rep., 443, 1
Markevitch M., Gonzalez A. H., David L., Vikhlinin A., Murray S., Forman W., Jones C., Tucker W., 2002, ApJ, 567, L27

Markevitch M., Govoni F., Brunetti G., Jerius D., 2005, ApJ, 627, 733

Mazzotta P., Bourdin H., Giacintucci S., Markevitch M., Venturi T., 2011, Mem. Soc. Astron. Ital., 82, 495

Miley G., 1980, ARA\&A, 18, 165

Miyashita Y., Ideguchi S., Takahashi K., 2016, PASJ, 68, 44

Offringa A. R., Smirnov O., 2017, MNRAS, 471, 301

Offringa A. R. et al., 2014, MNRAS, 444, 606

Oppermann N. et al., 2012, A\&A, 542, A93

Perley R. A., Butler B. J., 2013, ApJS, 206, 16

Petrosian V., 2001, ApJ, 557, 560

Piffaretti R., Arnaud M., Pratt G. W., Pointecouteau E., Melin J. B., 2011, A\&A, 534, A109

Pinzke A., Oh S. P., Pfrommer C., 2013, MNRAS, 435, 1061

Planck Collaboration XXVII, 2016, A\&A, 594, A27

Rajpurohit K. et al., 2018, ApJ, 852, 65

Rau U., Cornwell T. J., 2011, A\&A, 532, A71

Roettiger K., Burns J. O., Stone J. M., 1999, ApJ, 518, 603

Rossetti M. et al., 2016, MNRAS, 457, 4515

Rybicki G. B., Lightman A. P., 1986, Radiative Processes in Astrophysics. Wiley, New York

Schlickeiser R., Vainio R., Böttcher M., Lerche I., Pohl M., Schuster C., 2002, A\&A, 393, 69

Schnitzeler D. H. F. M., 2018, MNRAS, 474, 300

Schnitzeler D. H. F. M., Lee K. J., 2017, MNRAS, 466, 378

Snowden S. L., Mushotzky R. F., Kuntz K. D., Davis D. S., 2008, A\&A, 478,615

Sokoloff D. D., Bykov A. A., Shukurov A., Berkhuijsen E. M., Beck R., Poezd A. D., 1998, MNRAS, 299, 189

Subramanian K., 2016, Rep. Prog. Phys., 79, 076901

Sun X. H. et al., 2015, AJ, 149, 60

Taylor A. R., Stil J. M., Sunstrum C., 2009, ApJ, 702, 1230

Trasatti M., Akamatsu H., Lovisari L., Klein U., Bonafede A., Brüggen M., Dallacasa D., Clarke T., 2015, A\&A, 575, A45

Urdampilleta I., Akamatsu H., Mernier F., Kaastra J. S., de Plaa J., Ohashi T., Ishisaki Y., Kawahara H., 2018, A\&A, 618, A74

Valtchanov I., Murphy T., Pierre M., Hunstead R., Lémonon L., 2002, A\&A, 392, 795

van Weeren R. J. et al., 2012, A\&A, 543, A43

van Weeren R. J. et al., 2016, ApJ, 818, 204

van Weeren R. J. et al., 2017a, Nat. Astron., 1, 0005

van Weeren R. J. et al., 2017b, ApJ, 835, 197

van Weeren R. J., de Gasperin F., Akamatsu H., Brüggen M., Feretti L., Kang H., Stroe A., Zandanel F., 2019, Space Sci. Rev., 215, 16

Vazza F., Brüggen M., 2014, MNRAS, 437, 2291

Vazza F., Brunetti G., Gheller C., 2009, MNRAS, 395, 1333

Vazza F., Eckert D., Brüggen M., Huber B., 2015, MNRAS, 451, 2198

Vazza F., Brüggen M., Wittor D., Gheller C., Eckert D., Stubbe M., 2016, MNRAS, 459, 70

Vazza F., Brunetti G., Brüggen M., Bonafede A., 2018, MNRAS, 474, 1672

Venturi T., Giacintucci S., Brunetti G., Cassano R., Bardelli S., Dallacasa D., Setti G., 2007, A\&A, 463, 937

Venturi T., Giacintucci S., Dallacasa D., Cassano R., Brunetti G., Macario G., Athreya R., 2013, A\&A, 551, A24

Werner N., Urban O., Simionescu A., Allen S. W., 2013, Nature, 502, 656

Wittman D., Cornell B. H., Nguyen J., 2018, ApJ, 862, 160

Wittor D., Vazza F., Brüggen M., 2017, MNRAS, 464, 4448

Wright E. L., 2006, PASP, 118, 1711

This paper has been typeset from a $\mathrm{T}_{\mathrm{E} X / \mathrm{LT} \mathrm{E}} \mathrm{X}$ file prepared by the author. 